
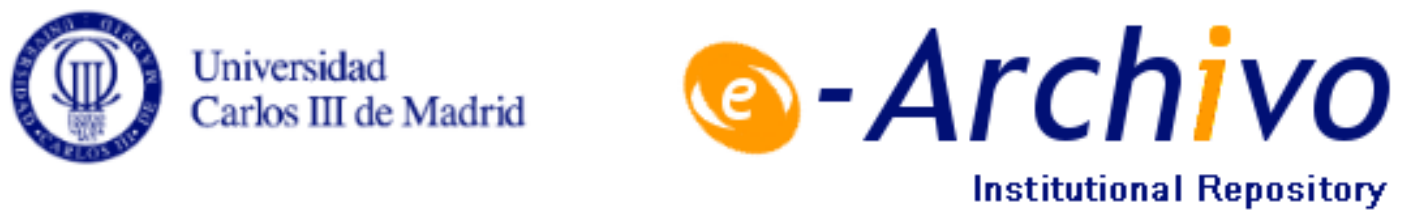

This is a postprint version of the following published document:

Dopico, F. M. and K. Pomés. Structured eigenvalue condition numbers for parameterized quasiseparable matrices, in Numerische Mathematik, 2015, 16 November. Available in http://dx.doi.org/10.1007/s00211-015-0779-5

(c) Springer-Verlag Berlin Heidelberg. 2015. 


\title{
Structured eigenvalue condition numbers for parameterized quasiseparable matrices
}

\author{
Froilán M. Dopico* Kenet Pomés*
}

October 10, 2015

\begin{abstract}
The development of fast algorithms for performing computations with $n \times n$ low-rank structured matrices has been a very active area of research during the last two decades, as a consequence of the numerous applications where these matrices arise. The key ideas behind these fast algorithms are that low-rank structured matrices can be described in terms of $O(n)$ parameters and that these algorithms operate on the parameters instead on the matrix entries. Therefore, the sensitivity of any computed quantity should be measured with respect to the possible variations that the parameters defining these matrices may suffer, since this determines the maximum accuracy of a given fast computation. In other words, it is necessary to develop condition numbers with respect to parameters for different magnitudes and classes of low-rank structured matrices, but, as far as we know, this has not yet been accomplished in any case. In this paper, we derive structured relative eigenvalue condition numbers for the important class of low-rank structured matrices known as $\{1 ; 1\}$-quasiseparable matrices with respect to relative perturbations of the parameters in the quasiseparable and in the Givens-vector representations of these matrices, and we provide fast algorithms for computing them. Comparisons among the new structured condition numbers and the unstructured one are also presented, as well as numerical experiments showing that the structured condition numbers can be small in situations where the unstructured one is huge. In addition, the approach presented in this paper is general and may be extended to other problems and classes of low-rank structured matrices.
\end{abstract}

Key words. condition numbers, simple eigenvalues, low-rank structured matrices, quasiseparable matrices, quasiseparable representation, Givens-vector representation.

AMS subject classification. 65F15, 65F $35,15 \mathrm{~A} 12,15 \mathrm{~A} 18$

\section{Introduction}

In simple words, a low-rank structured matrix is a matrix such that large submatrices of it have ranks much smaller than the size of the matrix. Perhaps, the best known examples of low-rank structured matrices are tridiagonal and other banded matrices with small bandwidth, for which all the submatrices lying in the (strictly) lower or upper triangular parts have ranks smaller than or equal to the bandwidth. These examples correspond to special cases of sparse matrices, but many other classes of dense lowrank structured matrices are available in the literature and arise in many applications. Research on low-rank structured matrices has received much attention in the last 15 years from the points of view of theory, computations, and applications. In fact, a number of recent books are devoted to this subject [19, 20, 42, 43], as well as survey papers [13], and the interested reader can find a huge number of references on this topic in them. From a numerical perspective, the key features of $n \times n$ low-rank structured matrices are that they can be very often described in terms of different sets of $O(n)$ parameters, called representations [42, Ch. 2], and that this fact has been used to develop many fast algorithms operating on these parameters to perform computations with low-rank structured matrices $[19,20,42,43]$. In this context, fast algorithms mean algorithms with cost $O(n)$ operations for solving linear systems of equations or with cost $O\left(n^{2}\right)$ operations for solving eigenvalue problems, which should be compared with the $O\left(n^{3}\right)$ cost of traditional dense matrix algorithms [26, 28].

\footnotetext{
*Departamento de Matemáticas, Universidad Carlos III de Madrid, Avda. Universidad 30, 28911 Leganés, Spain (dopico@math.uc3m.es, kpomes@math.uc3m.es). This research was partially supported by Ministerio de Economía y Competitividad of Spain through grant MTM2012-32542.
} 
Besides being the subject of modern research, low-rank structured matrices have an old and long history. One of the first examples of low-rank structured matrices are the single-pair matrices presented in 1941 in [24] in the context of totally nonnegative matrices (see also [23]). Another historical source of low-rank structured matrices is related to the efforts made in the 1950s to compute inverses of tridiagonal and, in general, of banded matrices with small bandwidth [1, 2, 7, 37]. These efforts were motivated by early research on the numerical solution of certain integral equations, boundary value problems, and problems in statistics. Inverses of banded matrices are included in a class of low-rank structured matrices called nowadays semiseparable matrices [42, Theorems 1.38 and 8.45]. Since the 1950s, the number of publications on low-rank structured matrices has increased considerably and, in fact, has exploded in the last 15 years. We refer the reader to the historical notes in $[19,20,42,43]$ and the detailed bibliography in [41].

Many interesting applications of low-rank structured matrices are discussed in the general references $[13,19,20,42,43]$, but here we would like to emphasize a few of them and to cite a few specific references as a sample. Fast computations with low-rank structured matrices have been used, for instance, in the numerical solution of elliptic partial differential equations [5, 27], in the numerical solution of integral equations $[12,33,34]$, and in the classical problem of computing all the roots of a polynomial of degree $n$ via matrix eigenvalue algorithms with cost of $O\left(n^{2}\right)$ operations and $O(n)$ storage $[9,11,14,15,21,39]$. With respect to this last problem, the recent reference [3] deserves special attention, since it includes a new algorithm and, for the first time in the literature, a rigorous proof that a fast and memory efficient algorithm for computing all the roots of a polynomial is backward stable in a matrix sense, which solves a long-standing open problem in Numerical Linear Algebra.

An important drawback of fast algorithms for low-rank structured matrices is that they have not been proved to be backward stable, with the exception of the particular ones in $[3,6,16]$. Taking into account the large number of references available on these algorithms, this lack of error analyses is striking. Possible reasons for it are that these fast algorithms are often involved, which makes the potential errors analyses very difficult (see the analysis in [16]) and, also, that some of them are potentially unstable in rare cases. In this scenario, a practical option is to estimate a posteriori error bounds for the outputs of these algorithms based on the classical approach in Numerical Linear Algebra of computing the residuals of the computed quantities, which give the backward errors, and multiply them by the corresponding condition numbers [26, 28, 29]. Since fast algorithms for low-rank structured matrices operate on parameters and not on matrix entries, the most sensible approach would be to estimate from the residuals the backward errors in the parameters defining the matrix, and to multiply them by the corresponding condition numbers with respect to perturbations of those parameters. The results in this paper are a first step in this ambitious plan, since we present for the first time in the literature condition numbers with respect to parameters for a family of low-rank structured matrices. More precisely, we develop eigenvalue condition numbers and show that some eigenvalues may be extremely ill-conditioned under general componentwise relative unstructured perturbations of the matrix entries, but very well-conditioned under perturbations in the parameters.

There exist many classes of low-rank structured matrices and it is not possible to cover all of them in this work. Therefore, we restrict ourselves to the particular but important class of $\{1 ; 1\}$-quasiseparable matrices, whose definition is recalled in Section 3. This class of matrices was introduced in [17] and includes several other relevant classes of low-rank structured matrices, as is discussed in [42, p. 10]. In addition, we would like to emphasize that the approach presented in this work can be easily extended to other classes of structured matrices as long as they are explicitly described in terms of parameters, as a consequence of the general framework developed in Section 2. So, we expect that the results in this paper can show how to get in the future condition numbers for many other classes of low-rank structured matrices and problems, as well as to foster more research on this topic.

This paper can also be seen as a new contribution to structured eigenvalue perturbation theory, a very fruitful and active area of research inside Numerical Linear Algebra. The general goal of the research in this area is to show that either for matrices in certain classes or for perturbations with particular properties, it is possible to derive much stronger eigenvalue perturbation bounds than the traditional ones obtained for general unstructured perturbations, and that these strong bounds can be used to prove that certain algorithms taking advantage of the structure yield much more accurate outputs than standard eigenvalue algorithms. The number of publications in this area is also very large and, here, we simply list a small sample of relevant references [22, 29, 30, 31, 32]. A common thread in structured eigenvalue perturbation theory is that the relative, instead the absolute, sensitivity of the eigenvalues is studied and bounded, as a consequence of the high expectations of the computations in our times. In addition, for the same reasons, many works on structured eigenvalue perturbation theory consider relative 
componentwise perturbations of the parameters defining the matrices. We follow both approaches in this paper, which are also motivated by the fact that the parameters defining a given quasiseparable matrix can be widely scaled, while yielding the same matrix [42, Chs. 1 \& 2], and so their collective norm is not related to the norm of the matrix. The results in this paper are, in particular, influenced by the recent ones in [22], but also influenced by the classical and seminal reference [36], which is often forgotten and which initiated the use of differential calculus for getting condition numbers.

Another goal of this paper is to provide a way to compare different representations of low-rank structured matrices. It is well known that the same quasiseparable matrix can be represented by different sets of parameters ([18], [42, Ch. 2]), also called generators, and it is not clear which set is more appropriate for developing a fast algorithm. A sensible option is to choose that representation for which the condition number of the desired quantity with respect to perturbations of the parameters is the smallest one. For this reason, we study and compare eigenvalue condition numbers for different representations of $\{1 ; 1\}$-quasiseparable matrices. More precisely, we consider all the quasiseparable representations [18], there are infinitely many, and the, essentially unique, Givens-vector representation ([40], [42, Ch. 2]), and we prove that the eigenvalue condition numbers have similar magnitudes for all of them, but that the one corresponding to the Givens-vector representation is the smallest. We advance that the most basic reason for this fact is the presence of extra constraints in the parameters of the Givens-vector representation with respect to the ones of the quasiseparable representation, which restrict the set of possible perturbations. In this context, it should be stressed that relative condition numbers do not take into account other issues which are also very important in practical computations, as the appearance of very large or small parameters that can produce overflow or underflow and spoil the whole computation.

Two remarkable unexpected properties are proved in this paper for the eigenvalue condition numbers of $\{1 ; 1\}$-quasiseparable matrices with respect to the (infinitely many) quasiseparable representations. First, that these condition numbers are independent of the particular representation (see Proposition 4.5) and, second, that they can be expressed just in terms of the matrix entries, i.e., without using any parametrization of the matrix (see Theorem 4.4). Nevertheless, the low-rank structure of the matrix is reflected in the way the different entries of the matrix contribute to the condition number. These properties are important because it is not always trivial to compute a parametrization of a low-rank structured matrix.

The rest of the paper is organized as follows. Section 2 presents the general results on eigenvalue condition numbers with respect to parameters that will be used throughout the paper. Section 3 recalls the notions of quasiseparable matrices and representations. Sections 4, 5, and 6 include the most important results in this paper on eigenvalue condition numbers of $\{1 ; 1\}$-quasiseparable matrices in the quasiseparable and Givens-vector representations, on fast algorithms with cost $O(n)$ flops for computing them, and on the comparison between them. Numerical experiments are presented in Section 7 and conclusions and lines of future research are established in Section 8.

Notation. We will follow a common notation in Numerical Linear Algebra and use capital Roman letters $A, B, \ldots$, for matrices, lower case Roman letters $\boldsymbol{x}, \boldsymbol{y}, \ldots$ for column vectors, and Greek letters $\alpha, \beta, \ldots$, for scalars. Except in the preliminary Section 2, only real matrices are considered, but some eigenvalues and eigenvectors may be complex. Given a complex column vector $\boldsymbol{y}$ of size $n \times 1, \boldsymbol{y}^{T}$ denotes its transpose, and $\boldsymbol{y}^{*}:=(\overline{\boldsymbol{y}})^{T}$ its conjugate transpose, where $\bar{\alpha}$ is the conjugate of $\alpha$ and conjugation of vectors should be understood in a componentwise sense. We consider the following usual norms

$$
\|\boldsymbol{y}\|_{1}:=\sum_{i=1}^{n}\left|y_{i}\right|, \quad\|\boldsymbol{y}\|_{2}:=\left(\sum_{i=1}^{n}\left|y_{i}\right|^{2}\right)^{\frac{1}{2}}, \text { and } \quad\|\boldsymbol{y}\|_{\infty}:=\max _{1 \leq i \leq n}\left|y_{i}\right|,
$$

where $y_{i}$ denotes the $i$-th component of $\boldsymbol{y}$, and the corresponding operator norms for matrices [26, 28]. For any square matrix $M$, we write the eigenvalue-eigenvector equations as $M \boldsymbol{x}=\lambda \boldsymbol{x}$ and $\boldsymbol{y}^{*} M=\lambda \boldsymbol{y}^{*}$, where $\boldsymbol{y}$ and $\boldsymbol{x}$ denote, respectively, the left and right eigenvectors associated to the eigenvalue $\lambda$ of $M$.

\section{Basics on eigenvalue condition numbers}

In this section we will present some well-known and some not so well-known results about eigenvalue condition numbers that are fundamental in this work. We will only consider simple eigenvalues since for a simple eigenvalue $\lambda$ with left and right eigenvectors $\boldsymbol{y}$ and $\boldsymbol{x}$ respectively, we have $\boldsymbol{y}^{*} \boldsymbol{x} \neq 0$. Theorem 2.1 and its Corollary 2.2 can be found in [38]. They are the fundamental results from which all the other results in this section are derived. 
The results in this section are valid for complex matrices. Note that any perturbation of a matrix $M \in \mathbb{C}^{n \times n}$ can be expressed as the sum $M+\delta M$, where $\delta M \in \mathbb{C}^{n \times n}$ is called the perturbation matrix.

Theorem 2.1. Let $\lambda$ be a simple eigenvalue of $M \in \mathbb{C}^{n \times n}$, with left and right eigenvectors $\boldsymbol{y}$ and $\boldsymbol{x}$, respectively. Then, for any matrix $\delta M \in \mathbb{C}^{n \times n}$, there is a unique eigenvalue $\tilde{\lambda}$ of $M+\delta M$ such that

$$
\tilde{\lambda}=\lambda+\frac{\boldsymbol{y}^{*}(\delta M) \boldsymbol{x}}{\boldsymbol{y}^{*} \boldsymbol{x}}+\mathcal{O}\left(\|\delta M\|^{2}\right)
$$

where $\|\delta M\|$ is any norm of $\delta M$.

Corollary 2.2. Let $\lambda$ be a simple eigenvalue of $M \in \mathbb{C}^{n \times n}$ with left and right eigenvectors $\boldsymbol{y}=$ $\left(y_{1}, \ldots, y_{n}\right)^{T}$ and $\boldsymbol{x}=\left(x_{1}, \ldots, x_{n}\right)^{T}$, respectively. Then $\lambda$ is a differentiable function of the entries $m_{i j}$ of $M$. Moreover,

$$
\frac{\partial \lambda}{\partial m_{i j}}(M)=\frac{\bar{y}_{i} x_{j}}{\boldsymbol{y}^{*} \boldsymbol{x}}
$$

On the other hand, in 1965, in [44], Wilkinson defined the notion of a condition number for simple eigenvalues. In the modern notation used, for instance, in [29], the Wilkinson condition number is defined as in Definition 2.3.

Definition 2.3. Let $\lambda$ be a simple eigenvalue of $M \in \mathbb{C}^{n \times n}$. Then the Wilkinson condition number of $\lambda$, denoted by $\kappa_{\lambda}$, is defined as

$$
\kappa_{\lambda}:=\lim _{\eta \rightarrow 0} \sup \left\{\frac{|\delta \lambda|}{\eta}:(\lambda+\delta \lambda) \text { is an eigenvalue of }(M+\delta M),\|\delta M\|_{2} \leq \eta\right\} .
$$

Based on this definition, if a left eigenvector and a right eigenvector of a simple eigenvalue $\lambda$ of $M \in \mathbb{C}^{n \times n}$ are known, it is easy to compute the Wilkinson condition number of $\lambda[29]$.

Theorem 2.4. Let $\lambda$ be a simple eigenvalue of $M \in \mathbb{C}^{n \times n}$, with left eigenvector $\boldsymbol{y} \in \mathbb{C}^{n}$ and right eigenvector $\boldsymbol{x} \in \mathbb{C}^{n}$. Then

$$
\kappa_{\lambda}=\frac{\|\boldsymbol{y}\|_{2}\|\boldsymbol{x}\|_{2}}{\left|\boldsymbol{y}^{*} \boldsymbol{x}\right|}=\frac{1}{\cos \angle(\boldsymbol{y}, \boldsymbol{x})}
$$

It is obvious, from its definition, that the Wilkinson condition number is an absolute-absolute normwise condition number, this means that it measures the absolute sensitivity of a simple eigenvalue with respect to absolute normwise perturbations of the matrix. In [10] the standard Wilkinson condition number was replaced by a relative-relative condition number, which means a relative measure with respect to relative normwise perturbations of the matrix.

Definition 2.5. Let $\lambda \neq 0$ be a simple eigenvalue of $M \in \mathbb{C}^{n \times n}$. Then we denote by $\kappa_{\lambda}^{\text {rel }}$ the relative Wilkinson condition number of $\lambda$ defined as

$$
\kappa_{\lambda}^{\mathrm{rel}}:=\lim _{\eta \rightarrow 0} \sup \left\{\frac{|\delta \lambda|}{\eta|\lambda|}:(\lambda+\delta \lambda) \text { is an eigenvalue of }(M+\delta M),\|\delta M\|_{2} \leq \eta\|M\|_{2}\right\} \text {. }
$$

Theorem 2.6. Let $\lambda \neq 0$ be a simple eigenvalue of $M \in \mathbb{C}^{n \times n}$ with left eigenvector $\boldsymbol{y} \in \mathbb{C}^{n}$ and right eigenvector $\boldsymbol{x} \in \mathbb{C}^{n}$. Then

$$
\kappa_{\lambda}^{\mathrm{rel}}=\frac{\|\boldsymbol{y}\|_{2}\|\boldsymbol{x}\|_{2}}{\left|\boldsymbol{y}^{*} \boldsymbol{x}\right|} \frac{\|M\|_{2}}{|\lambda|}=\kappa_{\lambda} \frac{\|M\|_{2}}{|\lambda|}
$$

Following the ideas in [22], we will use here a relative-relative componentwise condition number, that is, a measure of the relative variation of an eigenvalue with respect to the largest relative perturbation of each of the nonzero entries of the matrix. We denote by $|M| \in \mathbb{C}^{n \times n}$ the matrix whose entries are the absolute values of the entries of $M$ (i.e., $\left.|M|_{i j}:=\left|M_{i j}\right|\right)$ and we adopt a similar notation for vectors.

Definition 2.7. Let $\lambda \neq 0$ be a simple eigenvalue of $M \in \mathbb{C}^{n \times n}$. We define the relative componentwise condition number of $\lambda$ as

$$
\operatorname{cond}(\lambda ; M):=\lim _{\eta \rightarrow 0} \sup \left\{\frac{|\delta \lambda|}{\eta|\lambda|}:(\lambda+\delta \lambda) \text { is an eigenvalue of }(M+\delta M),|\delta M| \leq \eta|M|\right\} .
$$


The next theorem, stated for the first time in [25], gives an expression for computing $\operatorname{cond}(\lambda ; M)$ and it can be seen as a consequence of the more general Theorem 2.13 we prove later, so we present its proof at the end of this section.

Theorem 2.8. Let $\lambda \neq 0$ be a simple eigenvalue with left eigenvector $\boldsymbol{y}$ and right eigenvector $\boldsymbol{x}$ of the matrix $M \in \mathbb{C}^{n \times n}$. Then

$$
\operatorname{cond}(\lambda ; M)=\frac{\left|\boldsymbol{y}^{*}\right||M||\boldsymbol{x}|}{|\lambda|\left|\boldsymbol{y}^{*} \boldsymbol{x}\right|} .
$$

A useful property that is easy to prove about this condition number is that $\operatorname{cond}(\lambda ; M) \leq \sqrt{n} \kappa_{\lambda}^{\mathrm{rel}}$, and, in many important situations, $\operatorname{cond}(\lambda ; M)$ can be much smaller than $\kappa_{\lambda}^{\text {rel }}$.

Another important fact about $\operatorname{cond}(\lambda ; M)$ is that it is invariant under diagonal similarity while Wilkinson and relative Wilkinson condition numbers are not.

Lemma 2.9. For any scaling matrix $K$ invertible and diagonal,

$$
\operatorname{cond}\left(\lambda ; K M K^{-1}\right)=\operatorname{cond}(\lambda ; M) .
$$

Proof. Let $G=K M K^{-1}$. Note that if $\boldsymbol{y}$ and $\boldsymbol{x}$ are left and right eigenvectors of the matrix $M$ associated to the simple eigenvalue $\lambda$, then $\boldsymbol{y}_{K}^{*}=\boldsymbol{y}^{*} K^{-1}$ and $\boldsymbol{x}_{K}=K \boldsymbol{x}$ are the corresponding left and right eigenvectors of $G$ associated to $\lambda$. Furthermore, since $K$ is diagonal, no addition occurs in $K M K^{-1}$ and we have that $\left|K M K^{-1}\right|=|K||M|\left|K^{-1}\right|$. Consequently, $\left|\boldsymbol{y}_{K}^{*} \boldsymbol{x}_{K}\right|=\left|\boldsymbol{y}^{*} K^{-1} K \boldsymbol{x}\right|=\left|\boldsymbol{y}^{*} \boldsymbol{x}\right|$, and

$$
\operatorname{cond}(\lambda ; G)=\frac{\left|\boldsymbol{y}_{K}^{*}\right||G|\left|\boldsymbol{x}_{K}\right|}{|\lambda|\left|\boldsymbol{y}_{K}^{*} \boldsymbol{x}_{K}\right|}=\frac{\left|\boldsymbol{y}_{K}^{*}\right|\left|K M K^{-1}\right|\left|\boldsymbol{x}_{K}\right|}{|\lambda|\left|\boldsymbol{y}^{*} \boldsymbol{x}\right|}=\frac{\left|\boldsymbol{y}^{*}\right||M||\boldsymbol{x}|}{|\lambda|\left|\boldsymbol{y}^{*} \boldsymbol{x}\right|}=\operatorname{cond}(\lambda ; M) .
$$

Many interesting classes of matrices can be represented by sets of parameters different from its entries, whenever the entries are functions of certain parameters. Widely known examples include Cauchy, Vandermonde, and Toeplitz matrices [26, 28], among many others, and also the quasiseparable matrices considered in this work $[17,42]$. This motivates us to extend the definitions above to more general representations and to focus on relative componentwise eigenvalue condition numbers for representations.

Definition 2.10. Let $M \in \mathbb{C}^{n \times n}$ be a matrix whose entries are differentiable functions of a set of parameters $\Omega=\left(\omega_{1}, \omega_{2}, \ldots, \omega_{N}\right)^{T} \in \mathbb{C}^{N}$. This is denoted by $M(\Omega)$. Let $\lambda \neq 0$ be a simple eigenvalue of $M(\Omega)$ with left eigenvector $\boldsymbol{y}$ and right eigenvector $\boldsymbol{x}$. Then define

$$
\operatorname{cond}(\lambda, M ; \Omega):=\lim _{\eta \rightarrow 0} \sup \left\{\frac{|\delta \lambda|}{\eta|\lambda|}:(\lambda+\delta \lambda) \text { is an eigenvalue of } M(\Omega+\delta \Omega),|\delta \Omega| \leq \eta|\Omega|\right\} .
$$

If the matrix $M$ is clear from the context, then we will usually denote by $\operatorname{cond}(\lambda ; \Omega)$ the condition number $\operatorname{cond}(\lambda, M ; \Omega)$.

In order to find an explicit formula that allows us to calculate $\operatorname{cond}(\lambda ; \Omega)$, as it can be done with $\operatorname{cond}(\lambda ; M)$, the next definitions are convenient.

Definition 2.11. Let $M \in \mathbb{C}^{n \times n}$ be a matrix whose entries are differentiable functions of a set of parameters $\Omega=\left(\omega_{1}, \omega_{2}, \ldots, \omega_{N}\right)^{T} \in \mathbb{C}^{N}$. Let $\lambda \neq 0$ be a simple eigenvalue of $M(\Omega)$ with left eigenvector $\boldsymbol{y}$ and right eigenvector $\boldsymbol{x}$. We define the relative gradient of $\lambda$ with respect to $\Omega$ as the vector:

$$
\operatorname{relgrad}_{\Omega}(\lambda):=\left(\frac{\omega_{1}}{\lambda} \frac{\partial \lambda}{\partial \omega_{1}}, \ldots, \frac{\omega_{N}}{\lambda} \frac{\partial \lambda}{\partial \omega_{N}}\right)^{T}
$$

and the relative perturbation of $\Omega$ as the vector

$$
\operatorname{rel} \delta \Omega:=\left(\frac{\delta \omega_{1}}{\omega_{1}}, \ldots, \frac{\delta \omega_{N}}{\omega_{N}}\right)^{T},
$$

where if $w_{i}=0$ for some $i$, then we define $\delta w_{i} / w_{i} \equiv 0$ in agreement with Definition 2.10.

Taking into account our goals, the main result of this section is given in Theorem 2.13. But for proving that theorem, we will need the next proposition, which will also play an important role in calculating the componentwise relative eigenvalue condition number for quasiseparable matrices with respect to parameters. 
Proposition 2.12. Let $M \in \mathbb{C}^{n \times n}$ be a matrix whose entries are differentiable functions of a set of parameters $\Omega=\left(\omega_{1}, \omega_{2}, \ldots, \omega_{N}\right)^{T} \in \mathbb{C}^{N}$. This is denoted by $M(\Omega)$. Let $\lambda$ be a simple eigenvalue of $M(\Omega)$ with left eigenvector $\boldsymbol{y}$ and right eigenvector $\boldsymbol{x}$. Then

$$
\frac{\partial \lambda}{\partial \omega_{i}}=\frac{1}{\boldsymbol{y}^{*} \boldsymbol{x}}\left(\boldsymbol{y}^{*} \frac{\partial M(\Omega)}{\partial \omega_{i}} \boldsymbol{x}\right), \quad i \in\{1, \ldots, N\} .
$$

Proof. Compute explicitly the partial derivative of $M(\Omega) \boldsymbol{x}=\lambda \boldsymbol{x}$ to get

$$
\frac{\partial M(\Omega)}{\partial \omega_{i}} \boldsymbol{x}+M(\Omega) \frac{\partial \boldsymbol{x}}{\partial \omega_{i}}=\frac{\partial \lambda}{\partial \omega_{i}} \boldsymbol{x}+\lambda \frac{\partial \boldsymbol{x}}{\partial \omega_{i}},
$$

then, we multiply on the left the last equation by $\boldsymbol{y}^{*}$ and cancel out equal terms to find

$$
\boldsymbol{y}^{*} \frac{\partial M(\Omega)}{\partial \omega_{i}} \boldsymbol{x}=\frac{\partial \lambda}{\partial \omega_{i}} \boldsymbol{y}^{*} \boldsymbol{x},
$$

which completes the proof.

Theorem 2.13. Under the same hypotheses of Definition 2.10:

$$
\operatorname{cond}(\lambda ; \Omega)=\sum_{i=1}^{N}\left|\frac{\omega_{i}}{\lambda} \frac{\partial \lambda}{\partial \omega_{i}}\right|=\left\|\operatorname{relgrad}_{\Omega}(\lambda)\right\|_{1}
$$

and

$$
\frac{\omega_{i}}{\lambda} \frac{\partial \lambda}{\partial \omega_{i}}=\frac{1}{\lambda\left(\boldsymbol{y}^{*} \boldsymbol{x}\right)} \boldsymbol{y}^{*}\left(\omega_{i} \frac{\partial M(\Omega)}{\partial \omega_{i}}\right) \boldsymbol{x}, \quad \text { for } i=1, \ldots, N .
$$

Proof. We can form the absolute gradient vector by considering all the partial derivatives of $\lambda$ with respect to $\omega_{i}$ :

$$
\operatorname{grad}_{\Omega}(\lambda)=\left(\frac{\partial \lambda}{\partial \omega_{1}}, \ldots, \frac{\partial \lambda}{\partial \omega_{k}}, \ldots, \frac{\partial \lambda}{\partial \omega_{N}}\right)^{T},
$$

and, for infinitesimal absolute perturbations, $\delta \Omega:=\left(\delta \omega_{1}, \ldots, \delta \omega_{k}, \ldots, \delta \omega_{N}\right)^{T}$, we have

$$
\delta \lambda=\operatorname{grad}_{\Omega}(\lambda)^{T} \cdot \delta \Omega+\text { higher order terms (h.o.t). }
$$

Since $\delta \omega_{i}=0$ whenever $\omega_{i}=0$, following the convention in Definition 2.11, we rewrite (2.6) as

$$
\frac{\delta \lambda}{\lambda}=\left(\frac{\omega_{1}}{\lambda} \frac{\partial \lambda}{\partial \omega_{1}}, \ldots, \frac{\omega_{N}}{\lambda} \frac{\partial \lambda}{\partial \omega_{N}}\right) \cdot\left(\frac{\delta \omega_{1}}{\omega_{1}}, \ldots, \frac{\delta \omega_{N}}{\omega_{N}}\right)^{T}+\text { (h.o.t) }
$$

which can be rewritten in the notation from Definition 2.11 as

$$
\frac{\delta \lambda}{\lambda}=\operatorname{relgrad}_{\Omega}(\lambda)^{T} \cdot \operatorname{rel} \delta \Omega+(\text { h.o.t })
$$

Note that $|\delta \Omega| \leq \eta|\Omega|$ implies $|\operatorname{rel} \delta \Omega| \leq \eta(1,1, \ldots, 1)^{T}$, where $0<\eta \ll 1$. Thus, $\|\operatorname{rel} \delta \Omega\|_{\infty} \leq \eta$, and by applying the Hölder inequality $\left(\left|u^{T} v\right| \leq\|u\|_{1}\|v\|_{\infty}\right)$ in equation (2.8), we obtain

$$
\left.\left|\frac{\delta \lambda}{\lambda}\right| \leq\left\|\operatorname{relgrad}_{\Omega}(\lambda)\right\|_{1}\|\operatorname{rel} \delta \Omega\|_{\infty}+\text { (h.o.t. }\right) \leq \eta\left\|\operatorname{relgrad}_{\Omega}(\lambda)\right\|_{1}+\text { (h.o.t.). }
$$

From standard properties of norms (see [28, Ch. 6]), there exist particular vectors rel $\delta \Omega$ with infinity norm $\eta \operatorname{such}$ that $\left|\operatorname{relgrad}_{\Omega}(\lambda)^{T} \cdot \operatorname{rel} \delta \Omega\right|=\left\|\operatorname{relgrad}_{\Omega}(\lambda)\right\|_{1}\|\operatorname{rel} \delta \Omega\|_{\infty}$. Hence, for these vectors rel $\delta \Omega$, we have

$$
\left.\left|\frac{\delta \lambda}{\lambda}\right|=\left\|\operatorname{relgrad}_{\Omega}(\lambda)\right\|_{1}\|\operatorname{rel} \delta \Omega\|_{\infty}+\text { (h.o.t. }\right)=\eta\left\|\operatorname{relgrad}_{\Omega}(\lambda)\right\|_{1}+\text { (h.o.t.). }
$$

From (2.9), (2.10) and Definition 2.10 we prove immediately that if $\lambda \neq 0$, then (2.4) holds. Equation (2.5) follows from Proposition 2.12. 
Finally, we prove Theorem 2.8 as a particular case of Theorem 2.13, when the representation is given by the entries of $M$ themselves (i.e., $\left.\Omega=\left(m_{i j}\right)\right)$. In this case,

$$
m_{i j} \frac{\partial M}{\partial m_{i j}}=m_{i j} \boldsymbol{e}_{i} \boldsymbol{e}_{j}^{T}
$$

where $\boldsymbol{e}_{i}$ and $\boldsymbol{e}_{j}$ are the respective $i$ th and $j$ th canonical vectors in $\mathbb{C}^{n}$. Then, we can rewrite equations (2.5) and (2.4), respectively, as

$$
\begin{gathered}
\frac{m_{i j}}{\lambda} \frac{\partial \lambda}{\partial m_{i j}}=\frac{1}{\lambda\left(\boldsymbol{y}^{*} \boldsymbol{x}\right)} \bar{y}_{i} m_{i j} x_{j} \\
\operatorname{cond}(\lambda ; M)=\sum_{i=1, j=1}^{n} \frac{1}{|\lambda|\left|\left(\boldsymbol{y}^{*} \boldsymbol{x}\right)\right|}\left|\bar{y}_{i}\right|\left|m_{i j}\right|\left|x_{j}\right|=\frac{\left|\boldsymbol{y}^{*}\right||M \| \boldsymbol{x}|}{|\lambda|\left|\boldsymbol{y}^{*} \boldsymbol{x}\right|}
\end{gathered}
$$

which is Theorem 2.8 .

\section{Quasiseparable matrices}

Quasiseparable matrices were introduced for the first time in [17]. Before presenting the definition of a quasiseparable matrix, we need some additional notation. Given a matrix $A \in \mathbb{R}^{m \times n}$, we denote by $A(i: j, k: l)$, where $1 \leq i \leq j \leq m$ and $1 \leq k \leq l \leq n$, the submatrix of $A$ consisting of rows $i$ up to and including $j$ of $A$ and columns $k$ up to and including $l$ of $A$. This is the standard MATLAB notation for submatrices. The following definition can be found in [42, p. 301].

Definition 3.1 ( $\left\{n_{L} ; n_{U}\right\}$-quasiseparable matrix). A matrix $C \in \mathbb{R}^{n \times n}$ is called an $\left\{n_{L} ; n_{U}\right\}$-quasiseparable matrix, with $n_{L} \geq 0$ and $n_{U} \geq 0$, if the following two conditions are satisfied:

- every submatrix of $C$ entirely located in the strictly lower triangular part of $C$ has rank at most $n_{L}$, and there is at least one of these submatrices that has rank equal to $n_{L}$, and

- every submatrix of $C$ entirely located in the strictly upper triangular part of $C$ has rank at most $n_{U}$, and there is at least one of these submatrices that has rank equal to $n_{U}$.

This is obviously equivalent to: $\max _{i} \operatorname{rank} C(i+1: n, 1: i)=n_{L}$, and $\max _{i} \operatorname{rank} C(1: i, i+1: n)=n_{U}$.

A $\{1 ; 1\}$-quasiseparable matrix is often referred to as a $\{1\}$-quasiseparable matrix or simply as a quasiseparable matrix. These are the matrices that will be considered in this work.

\subsection{Representations}

Many classes of interesting matrices can be represented by a set of parameters different from the set of its entries. As one would expect, these representations are especially useful when they involve a much smaller number of parameters than the number of entries of the matrix. In some cases like banded matrices with small bandwidth, it is straightforward to find such a representation: take, for instance, the set of all the entries of the matrix that may be different from zero and organize them in a way that their positions in the matrix are known. But, in general, representations are not always easy to find. In Definition 3.2 (see [42, p. 56]) it is stated what is exactly meant by a representation of a class of matrices.

Definition 3.2. Let $\mathrm{V}$ and $\mathrm{W}$ be vector spaces containing the sets $\mathcal{V}$ and $\mathcal{W}$ respectively, and such that $\operatorname{dim}(\mathrm{V}) \leq \operatorname{dim}(\mathrm{W})$. An element $v \in \mathcal{V}$ is said to be a representation of another element $w \in \mathcal{W}$ if there exists a map $r: \mathcal{V} \longrightarrow \mathcal{W}$, such that $r(v)=w$, and $r(\mathcal{V})=\mathcal{W}$.

It is important to remark that the definition of a representation involves its existence not only for a particular element $w$ but for the whole given subset $\mathcal{W}$. In practical situations, the knowledge of a map $s: \mathcal{W} \longrightarrow \mathcal{V}$ such that the map $r \circ s=r(s): \mathcal{W} \longrightarrow \mathcal{W}$ is bijective and $r(s(w))=w, \forall w \in \mathcal{W}$, is also needed, since this map will allow us to obtain the desired representation for any element $w \in \mathcal{W}$. We also note from the previous definition that a representation of a given element $w \in \mathcal{W}$ (consider for instance a matrix in a given matrix class) may be not unique and, consequently, the choice of a representation for such class of matrices will heavily depend on criteria such as the number of parameters used by the representations and its stability with respect to the specific problem involving such matrices that we would like to solve, etc. An extensive description of different useful representations of low rank structured matrices, including quasiseparable matrices, can be found in [42, Ch. 2 and Sec. 8.5]. 


\section{Eigenvalue condition numbers for $\{1 ; 1\}$-quasiseparable matri- ces in the quasiseparable representation}

In this section we will deduce an expression for calculating the eigenvalue condition number for $\{1 ; 1\}$ quasiseparable matrices in the quasiseparable representation. This representation was introduced in [17], together with the definition of quasiseparable matrices, and will be described in Section 4.1. Sections 4.2 and 4.3 include the original results that we have obtained for these eigenvalue condition numbers. In these sections we establish the procedure and the main techniques that will be used through the rest of the work in order to obtain analogous results for the other condition numbers covered in this paper.

\subsection{The quasiseparable representation for $\{1 ; 1\}$-quasiseparable matrices}

Theorem 4.1, stated for $\{1 ; 1\}$-quasiseparable matrices, is a particular case of a theorem proved in [17] for $\left\{n_{L} ; n_{U}\right\}$-quasiseparable matrices and shows how any $\{1 ; 1\}$-quasiseparable matrix of size $n \times n$ can be represented with $\mathcal{O}(n)$ parameters instead of its $n^{2}$ entries.

Theorem 4.1. A matrix $C \in \mathbb{R}^{n \times n}$ is a $\{1 ; 1\}$-quasiseparable matrix if and only if it can be parameterized in terms of the following set of $7 n-8$ real parameters,

$$
\Omega_{Q S}=\left(\left\{p_{i}\right\}_{i=2}^{n},\left\{a_{i}\right\}_{i=2}^{n-1},\left\{q_{i}\right\}_{i=1}^{n-1},\left\{d_{i}\right\}_{i=1}^{n},\left\{g_{i}\right\}_{i=1}^{n-1},\left\{b_{i}\right\}_{i=2}^{n-1},\left\{h_{i}\right\}_{i=2}^{n}\right),
$$

as follows:

$$
C=\left[\begin{array}{rrrrr}
d_{1} & g_{1} h_{2} & g_{1} b_{2} h_{3} & \cdots & g_{1} b_{2} \ldots b_{n-1} h_{n} \\
p_{2} q_{1} & d_{2} & g_{2} h_{3} & \cdots & g_{2} b_{3} \ldots b_{n-1} h_{n} \\
p_{3} a_{2} q_{1} & p_{3} q_{2} & d_{3} & \cdots & g_{3} b_{4} \ldots b_{n-1} h_{n} \\
p_{4} a_{3} a_{2} q_{1} & p_{4} a_{3} q_{2} & p_{4} q_{3} & \cdots & g_{4} b_{5} \ldots b_{n-1} h_{n} \\
\vdots & \vdots & \vdots & \ddots & \vdots \\
p_{n} a_{n-1} a_{n-2} \ldots a_{2} q_{1} & p_{n} a_{n-1} \ldots a_{3} q_{2} & p_{n} a_{n-1} \ldots a_{4} q_{3} & \cdots & d_{n}
\end{array}\right],
$$

or, in a more compact notation,

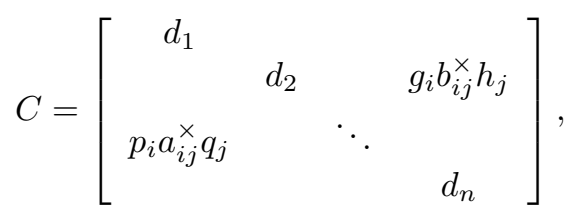

where $a_{i j}^{\times}=a_{i-1} a_{i-2} \cdots a_{j+1}$, for $i-1 \geq j+1, b_{i j}^{\times}=b_{i+1} b_{i+2} \cdots b_{j-1}$, for $i+1 \leq j-1, a_{j+1, j}^{\times}=1$, and $b_{j, j+1}^{\times}=1$ for $j=1, \ldots, n-1$.

Let us denote by $\mathrm{Q}^{n} \subset \mathbb{R}^{n \times n}$ the set of all $\{1 ; 1\}$-quasiseparable matrices of size $n \times n$. From Definition 3.2 and the previous theorem, the set of parameters $\Omega_{Q S}$ is a representation of the matrix $C \in \mathrm{Q}^{n}$ and therefore we call $\Omega_{Q S}$ a quasiseparable representation of $C$. Note that this representation is not unique as we can see in the following example.

Example 4.2. Let $C$ be a $\{1 ; 1\}$-quasiseparable matrix of size $5 \times 5$ and consider a quasiseparable representation of $C: \Omega_{Q S}=\left(\left\{p_{i}\right\}_{i=2}^{5},\left\{a_{i}\right\}_{i=2}^{4},\left\{q_{i}\right\}_{i=1}^{4},\left\{d_{i}\right\}_{i=1}^{5},\left\{g_{i}\right\}_{i=1}^{4},\left\{b_{i}\right\}_{i=2}^{4},\left\{h_{i}\right\}_{i=2}^{5}\right)$. Then,

$$
C=\left[\begin{array}{rrrrr}
d_{1} & g_{1} h_{2} & g_{1} b_{2} h_{3} & g_{1} b_{2} b_{3} h_{4} & g_{1} b_{2} b_{3} b_{4} h_{5} \\
p_{2} q_{1} & d_{2} & g_{2} h_{3} & g_{2} b_{3} h_{4} & g_{2} b_{3} b_{4} h_{5} \\
p_{3} a_{2} q_{1} & p_{3} q_{2} & d_{3} & g_{3} h_{4} & g_{3} b_{4} h_{5} \\
p_{4} a_{3} a_{2} q_{1} & p_{4} a_{3} q_{2} & p_{4} q_{3} & d_{4} & g_{4} h_{5} \\
p_{5} a_{4} a_{3} a_{2} q_{1} & p_{5} a_{4} a_{3} q_{2} & p_{5} a_{4} q_{3} & p_{5} q_{4} & d_{5}
\end{array}\right],
$$

and for every real number $\alpha \neq 0,1$, we also have

$$
C=\left[\begin{array}{rrrrr}
d_{1} & g_{1} h_{2} & g_{1} b_{2} h_{3} & g_{1} b_{2} b_{3} h_{4} & g_{1} b_{2} b_{3} b_{4} h_{5} \\
\left(\alpha p_{2}\right) \frac{q_{1}}{\alpha} & d_{2} & g_{2} h_{3} & g_{2} b_{3} h_{4} & g_{2} b_{3} b_{4} h_{5} \\
\left(\alpha p_{3}\right) a_{2} \frac{q_{1}}{\alpha} & \left(\alpha p_{3}\right) \frac{q_{2}}{\alpha} & d_{3} & g_{3} h_{4} & g_{3} b_{4} h_{5} \\
\left(\alpha p_{4}\right) a_{3} a_{2} \frac{q_{1}}{\alpha} & \left(\alpha p_{4}\right) a_{3} \frac{q_{2}}{\alpha} & \left(\alpha p_{4}\right) \frac{q_{3}}{\alpha} & d_{4} & g_{4} h_{5} \\
\left(\alpha p_{5}\right) a_{4} a_{3} a_{2} \frac{q_{1}}{\alpha} & \left(\alpha p_{5}\right) a_{4} a_{3} \frac{q_{2}}{\alpha} & \left(\alpha p_{5}\right) a_{4} \frac{q_{3}}{\alpha} & \left(\alpha p_{5}\right) \frac{q_{4}}{\alpha} & d_{5}
\end{array}\right]
$$


and we obtain a different quasiseparable representation of $C$ :

$$
\Omega_{Q S}^{\prime}=\left(\left\{\alpha p_{i}\right\}_{i=2}^{5},\left\{a_{i}\right\}_{i=2}^{4},\left\{q_{i} / \alpha\right\}_{i=1}^{4},\left\{d_{i}\right\}_{i=1}^{5},\left\{g_{i}\right\}_{i=1}^{4},\left\{b_{i}\right\}_{i=2}^{4},\left\{h_{i}\right\}_{i=2}^{5}\right) .
$$

Remark 4.3. There are many important subsets of $\{1 ; 1\}$-quasiseparable matrices arising in applications as, for instance, semiseparable matrices, generator representable semiseparable matrices, semiseparable plus diagonal matrices, and their corresponding symmetric versions [42, Ch. 1]. Although these particular subsets of matrices can be represented via the quasiseparable representation introduced in Theorem 4.1, they also admit other "more compressed" representations, i.e., in terms of less parameters, which are special instances of the quasiseparable representation. Such compressed representations can be found in [42, Chs. 1 and 2] and are the ones to be used in practice when working with these particular $\{1 ; 1\}$-quasiseparable matrices. The formalism presented in this paper can be directly applied to develop eigenvalue condition numbers with respect to these compressed representations. These condition numbers would reflect faithfully the particular structures of the subsets of matrices mentioned above and, therefore, would be smaller than the condition numbers developed here, since they restrict the possible perturbations in order to preserve the additional structures. For the sake of brevity, we do not develop such condition numbers in this paper.

\subsection{The eigenvalue condition number for $\{1 ; 1\}$-quasiseparable matrices in the quasiseparable representation: expression and properties}

From Example 4.2 it seems natural to consider relative componentwise perturbations of $\Omega_{Q S}$ instead of normwise perturbations of $\Omega_{Q S}$, because the norm of the vector of parameters does not determine the norm of the matrix. Since the matrix $C$ is differentiable with respect to these parameters, we can deduce eigenvalue relative-relative componentwise condition numbers for this parametrization by using Theorem 2.13 .

Theorem 4.4. Let $C \in \mathbb{R}^{n \times n}$ be a $\{1 ; 1\}$-quasiseparable matrix and let us express $C$ as $C=C_{L}+C_{D}+$ $C_{U}$, with $C_{L}$ strictly lower triangular, $C_{D}$ diagonal, and $C_{U}$ strictly upper triangular. Suppose $\lambda \neq 0$ is a simple eigenvalue of $C$ with left and right eigenvectors $\boldsymbol{y}$ and $\boldsymbol{x}$, respectively, and denote by $\Omega_{Q S}$ a quasiseparable representation of $C$. Then, the componentwise relative condition number cond $\left(\lambda ; \Omega_{Q S}\right)$ of $\lambda$ with respect to $\Omega_{Q S}$ is given by the following expression:

$$
\begin{aligned}
\operatorname{cond}\left(\lambda ; \Omega_{Q S}\right)= & \frac{1}{|\lambda|\left|\boldsymbol{y}^{*} \boldsymbol{x}\right|}\left\{\left|\boldsymbol{y}^{*}\right|\left|C_{D}\right||\boldsymbol{x}|+\left|\boldsymbol{y}^{*}\right|\left|C_{L} \boldsymbol{x}\right|+\left|\boldsymbol{y}^{*} C_{L}\right||\boldsymbol{x}|+\left|\boldsymbol{y}^{*}\right|\left|C_{U} \boldsymbol{x}\right|+\left|\boldsymbol{y}^{*} C_{U}\right||\boldsymbol{x}|\right. \\
& \left.+\sum_{i=2}^{n-1}\left|\boldsymbol{y}^{*}\left[\begin{array}{cc}
0 & 0 \\
C(i+1: n, 1: i-1) & 0
\end{array}\right] \boldsymbol{x}\right|+\sum_{j=2}^{n-1}\left|\boldsymbol{y}^{*}\left[\begin{array}{cc}
0 & C(1: j-1, j+1: n) \\
0 & 0
\end{array}\right] \boldsymbol{x}\right|\right\} .
\end{aligned}
$$

Proof. Let us consider $\Omega_{Q S}=\left(\left\{p_{i}\right\}_{i=2}^{n},\left\{a_{i}\right\}_{i=2}^{n-1},\left\{q_{i}\right\}_{i=1}^{n-1},\left\{d_{i}\right\}_{i=1}^{n},\left\{g_{i}\right\}_{i=1}^{n-1},\left\{b_{i}\right\}_{i=2}^{n-1},\left\{h_{i}\right\}_{i=2}^{n}\right)$, then, from Definition 2.11 and Theorem 2.13, we have $\operatorname{cond}\left(\lambda ; \Omega_{Q S}\right)=\left\|\operatorname{relgrad} \sin _{\Omega_{S}}(\lambda)\right\|_{1}$, where

$$
\begin{aligned}
\operatorname{relgrad}_{\Omega_{Q S}}(\lambda)= & \left(\frac{d_{1}}{\lambda} \frac{\partial \lambda}{\partial d_{1}}, \ldots, \frac{d_{n}}{\lambda} \frac{\partial \lambda}{\partial d_{n}}, \frac{p_{2}}{\lambda} \frac{\partial \lambda}{\partial p_{2}}, \ldots, \frac{p_{n}}{\lambda} \frac{\partial \lambda}{\partial p_{n}}, \frac{a_{2}}{\lambda} \frac{\partial \lambda}{\partial a_{2}}, \ldots, \frac{a_{n-1}}{\lambda} \frac{\partial \lambda}{\partial a_{n-1}}, \frac{q_{1}}{\lambda} \frac{\partial \lambda}{\partial q_{1}}, \ldots\right. \\
& \left.\frac{q_{n-1}}{\lambda} \frac{\partial \lambda}{\partial q_{n-1}}, \frac{g_{1}}{\lambda} \frac{\partial \lambda}{\partial g_{1}}, \ldots, \frac{g_{n-1}}{\lambda} \frac{\partial \lambda}{\partial g_{n-1}}, \frac{b_{2}}{\lambda} \frac{\partial \lambda}{\partial b_{2}}, \ldots, \frac{b_{n-1}}{\lambda} \frac{\partial \lambda}{\partial b_{n-1}}, \frac{h_{2}}{\lambda} \frac{\partial \lambda}{\partial h_{2}}, \ldots, \frac{h_{n}}{\lambda} \frac{\partial \lambda}{\partial h_{n}}\right)^{T} .
\end{aligned}
$$

Therefore, in order to find an explicit expression for $\left\|\operatorname{relgrad}_{\Omega_{Q S}}(\lambda)\right\|_{1}$, we will compute all the partial derivatives of $\lambda$ with respect to the parameters of $\Omega_{Q S}$ by using (2.3) from Proposition 2.12.

Derivatives with respect to $\left\{d_{i}\right\}_{i=1}^{n}$ : Since it is obvious that $\frac{\partial C}{\partial d_{i}}=\boldsymbol{e}_{i} \cdot \boldsymbol{e}_{i}^{T}$ (where $\boldsymbol{e}_{i}$ denotes the $i$ th canonical vector), we have

$$
\frac{\partial \lambda}{\partial d_{i}}=\frac{\left(\boldsymbol{y}^{*} \boldsymbol{e}_{i}\right)\left(\boldsymbol{e}_{i}^{T} \boldsymbol{x}\right)}{\boldsymbol{y}^{*} \boldsymbol{x}}=\frac{\bar{y}_{i} x_{i}}{\boldsymbol{y}^{*} \boldsymbol{x}}, \quad \text { and } \quad \frac{d_{i}}{\lambda} \frac{\partial \lambda}{\partial d_{i}}=\frac{1}{\lambda\left(\boldsymbol{y}^{*} \boldsymbol{x}\right)} \bar{y}_{i} d_{i} x_{i} .
$$

Derivatives with respect to $\left\{p_{i}\right\}_{i=2}^{n}$ : Taking into account that

$$
p_{i} \frac{\partial C}{\partial p_{i}}=\boldsymbol{e}_{i}\left[\begin{array}{llll}
C(i, 1: i-1) & 0 & \cdots & 0
\end{array}\right],
$$


we conclude that

$$
\frac{p_{i}}{\lambda} \frac{\partial \lambda}{\partial p_{i}}=\frac{1}{\lambda\left(\boldsymbol{y}^{*} \boldsymbol{x}\right)} \bar{y}_{i}\left[\begin{array}{llll}
C(i, 1: i-1) & 0 & \cdots & 0
\end{array}\right] \boldsymbol{x} ; \quad i=2: n .
$$

Derivatives with respect to $\left\{a_{i}\right\}_{i=2}^{n-1}$ : In this case it is also easy to see that

$$
\begin{gathered}
a_{i} \frac{\partial C}{\partial a_{i}}=\left[\begin{array}{cc}
0 & 0 \\
C(i+1: n, 1: i-1) & 0
\end{array}\right], \quad \text { and } \\
\frac{a_{i}}{\lambda} \frac{\partial \lambda}{\partial a_{i}}=\frac{1}{\lambda\left(\boldsymbol{y}^{*} \boldsymbol{x}\right)} \boldsymbol{y}^{*}\left[\begin{array}{cc}
0 & 0 \\
C(i+1: n, 1: i-1) & 0
\end{array}\right] \boldsymbol{x} ; \quad i=2: n-1 .
\end{gathered}
$$

Derivatives with respect to $\left\{q_{j}\right\}_{j=1}^{n-1}$ : Using that

$$
q_{j} \frac{\partial C}{\partial q_{j}}=\left[\begin{array}{c}
0 \\
C(j+1: n, j)
\end{array}\right] \boldsymbol{e}_{j}^{T}
$$

we obtain

$$
\frac{q_{j}}{\lambda} \frac{\partial \lambda}{\partial q_{j}}=\frac{1}{\lambda\left(\boldsymbol{y}^{*} \boldsymbol{x}\right)} \boldsymbol{y}^{*}\left[\begin{array}{c}
0 \\
C(j+1: n, j)
\end{array}\right] x_{j} ; \quad j=1: n-1 .
$$

In an analogous way we can find the partial derivatives of $\lambda$ with respect to the parameters $\left\{g_{i}\right\}_{i=1}^{n-1}$, $\left\{b_{i}\right\}_{i=2}^{n-1}$, and $\left\{h_{i}\right\}_{i=2}^{n}$, which describe the strictly upper triangular part of $C$.

Derivatives with respect to $\left\{g_{i}\right\}_{i=1}^{n-1}$ :

$$
\frac{g_{i}}{\lambda} \frac{\partial \lambda}{\partial g_{i}}=\frac{1}{\lambda\left(\boldsymbol{y}^{*} \boldsymbol{x}\right)} \bar{y}_{i}\left[\begin{array}{llll}
0 & \cdots & 0 & C(i, i+1: n)
\end{array}\right] \boldsymbol{x} ; \quad i=1: n-1 .
$$

Derivatives with respect to $\left\{b_{i}\right\}_{i=2}^{n-1}$ :

$$
\frac{b_{i}}{\lambda} \frac{\partial \lambda}{\partial b_{i}}=\frac{1}{\lambda\left(\boldsymbol{y}^{*} \boldsymbol{x}\right)}\left(\boldsymbol{y}^{*}\left[\begin{array}{cc}
0 & C(1: i-1, i+1: n) \\
0 & 0
\end{array}\right] \boldsymbol{x}\right) ; \quad i=2: n-1 .
$$

Derivatives with respect to $\left\{h_{j}\right\}_{j=2}^{n}$ :

$$
\frac{h_{j}}{\lambda} \frac{\partial \lambda}{\partial h_{j}}=\frac{1}{\lambda\left(\boldsymbol{y}^{*} \boldsymbol{x}\right)}\left(\boldsymbol{y}^{*}\left[\begin{array}{c}
C(1: j-1, j) \\
0
\end{array}\right] x_{j}\right) ; \quad j=2: n .
$$

Now it only remains to calculate $\left\|\operatorname{relgrad}_{\Omega_{Q S}}(\lambda)\right\|_{1}$ and we will proceed by parts using the decomposition $C=C_{L}+C_{D}+C_{U}$. We will denote by $C(i,:)$ the $i$ th row vector of the matrix $C$. Since the matrix $C_{L}$ is strictly lower triangular and the matrix $C_{U}$ is strictly upper triangular, the following equalities hold,

$$
\begin{aligned}
& {\left[\begin{array}{ll}
C(i, 1: i-1) & 0
\end{array}\right]=C_{L}(i,:), C_{L}(1,:)=0 ;\left[\begin{array}{c}
0 \\
C(j+1: n, j)
\end{array}\right]=C_{L}(:, j), C_{L}(:, n)=0} \\
& {\left[\begin{array}{ll}
0 & C(i, i+1: n)
\end{array}\right]=C_{U}(i,:), C_{U}(n,:)=0 ;\left[\begin{array}{c}
C(1: j-1, j) \\
0
\end{array}\right]=C_{U}(:, j), C_{U}(:, 1)=0 .}
\end{aligned}
$$


Finally, let us consider the following sums :

$$
\begin{aligned}
& K_{d}=\sum_{i=1}^{n}\left|\frac{d_{i}}{\lambda} \frac{\partial \lambda}{\partial d_{i}}\right|=\frac{1}{\left|\lambda\left(\boldsymbol{y}^{*} \boldsymbol{x}\right)\right|}\left|\boldsymbol{y}^{*}\right|\left|C_{D}\right||\boldsymbol{x}| ; \\
& K_{p}=\sum_{i=2}^{n}\left|\frac{p_{i}}{\lambda} \frac{\partial \lambda}{\partial p_{i}}\right|=\frac{1}{\left|\lambda\left(\boldsymbol{y}^{*} \boldsymbol{x}\right)\right|} \sum_{i=2}^{n}\left|\bar{y}_{i}\right|\left|C_{L}(i,:) \boldsymbol{x}\right|=\frac{1}{\left|\lambda\left(\boldsymbol{y}^{*} \boldsymbol{x}\right)\right|}\left|\boldsymbol{y}^{*}\right|\left|C_{L} \boldsymbol{x}\right| ; \\
& K_{a}=\sum_{i=2}^{n-1}\left|\frac{a_{i}}{\lambda} \frac{\partial \lambda}{\partial a_{i}}\right|=\frac{1}{\left|\lambda\left(\boldsymbol{y}^{*} \boldsymbol{x}\right)\right|} \sum_{i=2}^{n-1}\left|\boldsymbol{y}^{*}\left[\begin{array}{cc}
0 & 0 \\
C(i+1: n, 1: i-1) & 0
\end{array}\right] \boldsymbol{x}\right| \text {; } \\
& K_{q}=\sum_{j=1}^{n-1}\left|\frac{q_{j}}{\lambda} \frac{\partial \lambda}{\partial q_{j}}\right|=\frac{1}{\left|\lambda\left(\boldsymbol{y}^{*} \boldsymbol{x}\right)\right|} \sum_{j=1}^{n-1}\left|\boldsymbol{y}^{*} C_{L}(:, j)\right|\left|x_{j}\right|=\frac{1}{\left|\lambda\left(\boldsymbol{y}^{*} \boldsymbol{x}\right)\right|}\left|\boldsymbol{y}^{*} C_{L}\right||\boldsymbol{x}| ; \\
& K_{g}=\sum_{i=1}^{n-1}\left|\frac{g_{i}}{\lambda} \frac{\partial \lambda}{\partial g_{i}}\right|=\frac{1}{\left|\lambda\left(\boldsymbol{y}^{*} \boldsymbol{x}\right)\right|} \sum_{i=1}^{n-1}\left|y_{i}\right|\left|C_{U}(i,:) \boldsymbol{x}\right|=\frac{1}{\left|\lambda\left(\boldsymbol{y}^{*} \boldsymbol{x}\right)\right|}\left|\boldsymbol{y}^{*}\right|\left|C_{U} \boldsymbol{x}\right| ; \\
& K_{b}=\sum_{j=2}^{n-1}\left|\frac{b_{j}}{\lambda} \frac{\partial \lambda}{\partial b_{j}}\right|=\frac{1}{\left|\lambda\left(\boldsymbol{y}^{*} \boldsymbol{x}\right)\right|} \sum_{j=2}^{n-1}\left|\boldsymbol{y}^{*}\left[\begin{array}{cc}
0 & C(1: j-1, j+1: n) \\
0 & 0
\end{array}\right] \boldsymbol{x}\right| \text {; } \\
& K_{h}=\sum_{j=2}^{n}\left|\frac{h_{j}}{\lambda} \frac{\partial \lambda}{\partial h_{j}}\right|=\frac{1}{\left|\lambda\left(\boldsymbol{y}^{*} \boldsymbol{x}\right)\right|} \sum_{j=2}^{n}\left|\boldsymbol{y}^{*} C_{U}(:, j)\right|\left|x_{j}\right|=\frac{1}{\left|\lambda\left(\boldsymbol{y}^{*} \boldsymbol{x}\right)\right|}\left|\boldsymbol{y}^{*} C_{U}\right||\boldsymbol{x}| ;
\end{aligned}
$$

We complete this proof by observing that $\operatorname{cond}\left(\lambda ; \Omega_{Q S}\right)=K_{d}+K_{p}+K_{a}+K_{q}+K_{g}+K_{b}+K_{h}$.

The explicit formula given in Theorem 4.4 for $\operatorname{cond}\left(\lambda ; \Omega_{Q S}\right)$ does not depend on the parameters of the chosen quasiseparable representation; it only depends on the matrix entries, the simple eigenvalue $\lambda$, and the left and right eigenvectors. This important property allows us to state the following proposition.

Proposition 4.5. Let $C \in \mathbb{R}^{n \times n}$ be a $\{1 ; 1\}$-quasiseparable matrix and $\lambda \neq 0$ be a simple eigenvalue of $C$. Then, for any two sets $\Omega_{Q S}$ and $\Omega_{Q S}^{\prime}$ of quasiseparable parameters of $C$,

$$
\operatorname{cond}\left(\lambda ; \Omega_{Q S}\right)=\operatorname{cond}\left(\lambda ; \Omega_{Q S}^{\prime}\right) .
$$

Another important property for this relative componentwise condition number appears from the natural comparison with the unstructured relative entrywise condition number defined in Definition 2.7 and given in Theorem 2.8. This comparison is established in the next proposition.

Proposition 4.6. Let $C$ be a $\{1 ; 1\}$-quasiseparable matrix and consider a set of quasiseparable parameters $\Omega_{Q S}$ of $C$. Let $\lambda \neq 0$ be a simple eigenvalue of $C$. Then, the following relation holds,

$$
\operatorname{cond}\left(\lambda ; \Omega_{Q S}\right) \leq n \operatorname{cond}(\lambda ; C) \text {. }
$$

Proof. From Theorem 4.4, and standard inequalities of absolute values we get:

$$
\begin{aligned}
\operatorname{cond}\left(\lambda ; \Omega_{Q S}\right) \leq & \frac{1}{|\lambda|\left|\boldsymbol{y}^{*} \boldsymbol{x}\right|}\left\{\left|\boldsymbol{y}^{*}\right|\left|C_{D}\right||\boldsymbol{x}|+\left|\boldsymbol{y}^{*}\right|\left|C_{L}\right||\boldsymbol{x}|+\left|\boldsymbol{y}^{*}\right|\left|C_{L}\right||\boldsymbol{x}|+\left|\boldsymbol{y}^{*}\right|\left|C_{U}\right||\boldsymbol{x}|+\left|\boldsymbol{y}^{*}\right|\left|C_{U}\right||\boldsymbol{x}|\right. \\
& \left.+\sum_{i=2}^{n-1}\left|\boldsymbol{y}^{*}\right|\left|C_{L}\right||\boldsymbol{x}|+\sum_{j=2}^{n-1}\left|\boldsymbol{y}^{*}\right|\left|C_{U}\right||\boldsymbol{x}|\right\} \\
\leq & \frac{1}{|\lambda|\left|\boldsymbol{y}^{*} \boldsymbol{x}\right|}\left\{\left|\boldsymbol{y}^{*}\right|\left|C_{D}\right||\boldsymbol{x}|+n\left|\boldsymbol{y}^{*}\right|\left|C_{L}\right||\boldsymbol{x}|+n\left|\boldsymbol{y}^{*}\right|\left|C_{U}\right||\boldsymbol{x}|\right\} \leq n \frac{\left|\boldsymbol{y}^{*}\right||C||\boldsymbol{x}|}{\left|\lambda \| \boldsymbol{y}^{*} \boldsymbol{x}\right|}=n \operatorname{cond}(\lambda ; C) .
\end{aligned}
$$

According to Proposition 4.6, the structured condition number $\operatorname{cond}\left(\lambda ; \Omega_{Q S}\right)$ is smaller than the unstructured condition number $\operatorname{cond}(\lambda ; C)$, except for a factor of $n$, but it can be potentially much smaller, as we will see in our numerical experiments (see Section 7 ). The factor $n$ comes from the entries $C_{1 n}$ and $C_{n 1}$. For instance, $C_{n 1}=p_{n} a_{n-1} \cdots a_{2} q_{1}$, implies that an entrywise relative perturbation of size $\eta$ over the representation $\Omega_{Q S}$ will generate a perturbation on the entry $C_{n 1}$ of the matrix $C$ involving $n$ factors of the form $(1+\delta)$, where $\delta$ represents the relative perturbations on the parameters. 
On the other hand, the exact expression of $\operatorname{cond}\left(\lambda ; \Omega_{Q S}\right)$ deduced in Theorem 4.4 is complicated, especially because of the summations appearing in the last two terms. Surprisingly, these two summations can be removed in order to define the effective condition number introduced in Definition 4.7, which can be used to estimate $\operatorname{cond}\left(\lambda ; \Omega_{Q S}\right)$ reliably up to a factor $n$. This is proved in Proposition 4.8.

Definition 4.7. Under the same hypotheses of Theorem 4.4, we define the effective relative condition number cond $\mathrm{eff}\left(\lambda ; \Omega_{Q S}\right)$ of $\lambda$ with respect to the quasiseparable representation $\Omega_{Q S}$ of $C$ as,

$$
\operatorname{cond}_{\mathrm{eff}}\left(\lambda ; \Omega_{Q S}\right):=\frac{1}{|\lambda|\left|\boldsymbol{y}^{*} \boldsymbol{x}\right|}\left\{\left|\boldsymbol{y}^{*}\right|\left|C_{D}\right||\boldsymbol{x}|+\left|\boldsymbol{y}^{*}\right|\left|C_{L} \boldsymbol{x}\right|+\left|\boldsymbol{y}^{*} C_{L}\right||\boldsymbol{x}|+\left|\boldsymbol{y}^{*}\right|\left|C_{U} \boldsymbol{x}\right|+\left|\boldsymbol{y}^{*} C_{U}\right||\boldsymbol{x}|\right\} .
$$

Proposition 4.8. Let $C \in \mathbb{R}^{n \times n}$ be a $\{1 ; 1\}$-quasiseparable matrix with a simple eigenvalue $\lambda \neq 0$ with left and right eigenvectors $\boldsymbol{y}$ and $\boldsymbol{x}$, respectively. Let $\Omega_{Q S}$ be a quasiseparable representation of $C$. Then

$$
\operatorname{cond}_{\mathrm{eff}}\left(\lambda ; \Omega_{Q S}\right) \leq \operatorname{cond}\left(\lambda ; \Omega_{Q S}\right) \leq(n-1) \operatorname{cond}_{\mathrm{eff}}\left(\lambda ; \Omega_{Q S}\right) .
$$

Proof. The first inequality is trivial from the definitions of cond $\mathrm{eff}\left(\lambda ; \Omega_{Q S}\right)$ and cond $\left(\lambda ; \Omega_{Q S}\right)$, respectively. On the other hand, note that

$$
\begin{aligned}
\left|\boldsymbol{y}^{*}\left[\begin{array}{cc}
0 & 0 \\
C(i+1: n, 1: i-1) & 0
\end{array}\right] \boldsymbol{x}\right| & \leq\left|\boldsymbol{y}^{*}\left[\begin{array}{cc}
0 \\
C_{L}(i+1: n, 1: i-1) & C_{L}(i+1: n, i: n)
\end{array}\right] \boldsymbol{x}\right| \\
& +\left|\boldsymbol{y}^{*}\left[\begin{array}{cc}
0 & 0 \\
0 & -C_{L}(i+1: n, i: n)
\end{array}\right] \boldsymbol{x}\right| \\
& \leq\left|\boldsymbol{y}^{*}\right|\left|\left[\begin{array}{c}
0 \\
C_{L}(i+1: n,:)
\end{array}\right] \boldsymbol{x}\right|+\left|\boldsymbol{y}^{*}\left[\begin{array}{cc}
0 & 0 \\
0 & C_{L}(i+1: n, i: n)
\end{array}\right]\right||\boldsymbol{x}| \\
& \leq\left|\boldsymbol{y}^{*}\right|\left|C_{L} \boldsymbol{x}\right|+\left|\boldsymbol{y}^{*} C_{L}\right||\boldsymbol{x}|,
\end{aligned}
$$

from where we obtain:

$$
\sum_{i=2}^{n-1}\left|\boldsymbol{y}^{*}\left[\begin{array}{cc}
0 & 0 \\
C(i+1: n, 1: i-1) & 0
\end{array}\right] \boldsymbol{x}\right| \leq(n-2)\left|\boldsymbol{y}^{*}\right|\left|C_{L} \boldsymbol{x}\right|+(n-2)\left|\boldsymbol{y}^{*} C_{L}\right||\boldsymbol{x}| .
$$

In an analogous way, we can prove that

$$
\sum_{j=2}^{n-1}\left|\boldsymbol{y}^{*}\left[\begin{array}{cc}
0 & C(1: j-1, j+1: n) \\
0 & 0
\end{array}\right] \boldsymbol{x}\right| \leq(n-2)\left|\boldsymbol{y}^{*} C_{U}\right||\boldsymbol{x}|+(n-2)\left|\boldsymbol{y}^{*}\right|\left|C_{U} \boldsymbol{x}\right| .
$$

Finally, from (4.1) and (4.2) it is straightforward that

$$
\operatorname{cond}\left(\lambda ; \Omega_{Q S}\right) \leq \frac{n-1}{|\lambda|\left|\boldsymbol{y}^{*} \boldsymbol{x}\right|}\left\{\left|\boldsymbol{y}^{*}\right|\left|C_{D}\right||\boldsymbol{x}|+\left|\boldsymbol{y}^{*}\right|\left|C_{L} \boldsymbol{x}\right|+\left|\boldsymbol{y}^{*} C_{L}\right||\boldsymbol{x}|+\left|\boldsymbol{y}^{*}\right|\left|C_{U} \boldsymbol{x}\right|+\left|\boldsymbol{y}^{*} C_{U}\right||\boldsymbol{x}|\right\},
$$

which completes the proof.

Another important property of eigenvalue condition numbers that must be studied is their behavior under diagonal similarities since many algorithms for computing eigenvalues of matrices start by balancing the matrix, i.e., by performing a diagonal similarity that for each $i$ makes the norm $\left(\|\cdot\|_{1},\|\cdot\|_{2}\right.$, or $\left.\|\cdot\|_{\infty}\right)$ of the $i$ th row equal to the norm $\left(\|\cdot\|_{1},\|\cdot\|_{2}\right.$, or $\left.\|\cdot\|_{\infty}\right)$ of the $i t h$ column (see [35], [26, p. 360-361]). For such purpose, Lemmas 4.9 and 4.10 will be useful for proving Theorem 4.11 , which will establish the invariance of $\operatorname{cond}\left(\lambda ; \Omega_{Q S}\right)$ under diagonal similarities. In the following, we will denote by $K=\operatorname{diag}\left(k_{1}, k_{2}, \cdots, k_{n}\right)$ any diagonal matrix $K \in \mathbb{R}^{n \times n}$ such that $K_{i i}=k_{i}$. For any matrix $C$ and any two ordered sets $\mathcal{I}$ and $\mathcal{J}$, we will denote by $C(\mathcal{I}, \mathcal{J})$ the submatrix of $C$ consisting of rows and columns with indices in $\mathcal{I}$ and $\mathcal{J}$, respectively.

Lemma 4.9. Let $K=\operatorname{diag}\left(k_{1}, k_{2}, \cdots, k_{n}\right)$ be an invertible diagonal matrix, and let $A, B \in \mathbb{R}^{n \times n}$ be matrices such that $B=K A K^{-1}$. Then, the following assertions hold.

(a) For any two ordered subsets $\mathcal{I}_{p}=\left\{i_{1}, i_{2}, \cdots, i_{p}\right\}$ and $\mathcal{J}_{q}=\left\{j_{1}, j_{2}, \cdots, j_{q}\right\}$ of indices such that $1 \leq i_{1} \leq i_{2} \leq \cdots \leq i_{p} \leq n$ and $1 \leq j_{1} \leq j_{2} \leq \cdots \leq j_{q} \leq n$, we have that

$$
B\left(\mathcal{I}_{p}, \mathcal{J}_{q}\right)=\operatorname{diag}\left(k_{i_{1}}, k_{i_{2}}, \ldots, k_{i_{p}}\right) \cdot A\left(\mathcal{I}_{p}, \mathcal{J}_{q}\right) \cdot \operatorname{diag}\left(1 / k_{j_{1}}, 1 / k_{j_{2}}, \ldots, 1 / k_{j_{q}}\right) .
$$


(b) For any matrix $C \in \mathbb{R}^{n \times n}$, let us denote by $\tilde{C}_{i_{1: p}, j_{1: q}} \in \mathbb{R}^{n \times n}$ a matrix such that $\tilde{C}_{i_{1: p}, j_{1: q}}\left(\mathcal{I}_{p}, \mathcal{J}_{q}\right)=$ $C\left(\mathcal{I}_{p}, \mathcal{J}_{q}\right)$, and $\tilde{C}_{i_{1: p}, j_{1: q}}(i, j)=0$, for any other entry. Then $\tilde{B}_{i_{1: p}, j_{1: q}}=K \tilde{A}_{i_{1: p}, j_{1: q}} K^{-1}$.

(c) If we decompose the matrices $A=A_{L}+A_{D}+A_{U}$ and $B=B_{L}+B_{D}+B_{U}$, where $A_{L}$ and $B_{L}$ are strictly lower triangular matrices, $A_{D}$ and $B_{D}$ are diagonal matrices, and $A_{U}$ and $B_{U}$ are strictly upper triangular matrices, then $B_{L}=K A_{L} K^{-1}, B_{D}=K A_{D} K^{-1}=A_{D}$, and $B_{U}=K A_{U} K^{-1}$.

(d) A vector $\boldsymbol{x}_{A} \in \mathbb{R}^{n \times 1}$ is a right eigenvector of $A$ associated to the eigenvalue $\lambda$ if and only if the vector $\boldsymbol{x}_{B}=K \boldsymbol{x}_{A}$ is a right eigenvector of $B$ associated to $\lambda$. Similarly, a vector $\boldsymbol{y}_{A} \in \mathbb{R}^{n \times 1}$ is a left eigenvector of $A$ associated to $\lambda$ if and only if the vector $\boldsymbol{y}_{B}=\left(\boldsymbol{y}_{A}^{*} K^{-1}\right)^{*}$ is a left eigenvector of $B$ associated to $\lambda$.

Proof. The proofs of (a), (b), and (c) are straightforward. So, we only prove part (d). The result follows from the equivalences:

$$
\begin{aligned}
& A \boldsymbol{x}_{A}=\lambda \boldsymbol{x}_{A} \Leftrightarrow \quad K^{-1} B K \boldsymbol{x}_{A}=\lambda \boldsymbol{x}_{A} \Leftrightarrow B\left(K \boldsymbol{x}_{A}\right)=\lambda\left(K \boldsymbol{x}_{A}\right), \text { and } \\
& \boldsymbol{y}_{A}^{*} A=\lambda \boldsymbol{y}_{A}^{*} \Leftrightarrow \quad \boldsymbol{y}_{A}^{*} K^{-1} B K=\lambda \boldsymbol{y}_{A}^{*} \Leftrightarrow\left(\boldsymbol{y}_{A}^{*} K^{-1}\right) B=\lambda\left(\boldsymbol{y}_{A}^{*} K^{-1}\right) .
\end{aligned}
$$

Lemma 4.10. Let $K=\operatorname{diag}\left(k_{1}, k_{2}, \cdots, k_{n}\right)$ be an invertible diagonal matrix and $C \in \mathbb{R}^{n \times n}$ be $a\{1 ; 1\}$ quasiseparable matrix. Then, the following two assertions hold.

(a) The matrix $K C K^{-1}$ is also a $\{1 ; 1\}$-quasiseparable matrix.

(b) If the set of parameters $\Omega_{Q S}=\left(\left\{p_{i}\right\}_{i=2}^{n},\left\{a_{i}\right\}_{i=2}^{n-1},\left\{q_{i}\right\}_{i=1}^{n-1},\left\{d_{i}\right\}_{i=1}^{n},\left\{g_{i}\right\}_{i=1}^{n-1},\left\{b_{i}\right\}_{i=2}^{n-1},\left\{h_{i}\right\}_{i=2}^{n}\right)$ is a quasiseparable representation of $C$, then the set of parameters $\Omega_{Q S}^{\prime}=\left(\left\{k_{i} p_{i}\right\}_{i=2}^{n},\left\{a_{i}\right\}_{i=2}^{n-1},\left\{q_{i} / k_{i}\right\}_{i=1}^{n-1}\right.$, $\left.\left\{d_{i}\right\}_{i=1}^{n},\left\{k_{i} g_{i}\right\}_{i=1}^{n-1},\left\{b_{i}\right\}_{i=2}^{n-1},\left\{h_{i} / k_{i}\right\}_{i=2}^{n}\right)$, is a quasiseparable representation of $K C K^{-1}$.

Proof. (a) It follows from (a) in Lemma 4.9 that for any two subsets $\left\{i_{1}, i_{2}, \cdots, i_{p}\right\}$ and $\left\{j_{1}, j_{2}, \cdots, j_{q}\right\}$ of indices such that $1 \leq i_{1} \leq i_{2} \leq \cdots \leq i_{p} \leq n$ and $1 \leq j_{1} \leq j_{2} \leq \cdots \leq j_{q} \leq n$, we have that

$$
\operatorname{rank} K C K^{-1}\left(\left\{i_{1}, i_{2}, \cdots, i_{p}\right\},\left\{j_{1}, j_{2}, \cdots, j_{q}\right\}\right)=\operatorname{rank} C\left(\left\{i_{1}, i_{2}, \cdots, i_{p}\right\},\left\{j_{1}, j_{2}, \cdots, j_{q}\right\}\right),
$$

and the result follows from Definition 3.1.

(b) It follows from Theorem 4.1 and from $K C K^{-1}(i, j)=k_{i} C(i, j) \frac{1}{k_{j}}$.

Theorem 4.11. Let $C \in \mathbb{R}^{n \times n}$ be $\{1 ; 1\}$-quasiseparable, $\lambda \neq 0$ be a simple eigenvalue of $C, K \in \mathbb{R}^{n \times n}$ be diagonal and nonsingular, $\Omega_{Q S}$ be any set of quasiseparable parameters of $C$, and $\Omega_{Q S}^{\prime}$ be any set of quasiseparable parameters of $K C K^{-1}$. Then

$$
\operatorname{cond}\left(\lambda, C ; \Omega_{Q S}\right)=\operatorname{cond}\left(\lambda, K C K^{-1} ; \Omega_{Q S}^{\prime}\right) .
$$

Proof. Note first that for any two matrices $A \in \mathbb{R}^{n \times n}$ and $B \in \mathbb{R}^{n \times n}$ we have that $|A K||B|=$ $|A||K||B|=|A||K B|$, since $K$ is diagonal. Let us consider $B=K C K^{-1}$ and let us analyze the expression given in Theorem 4.4 for cond $\left(\lambda, C ; \Omega_{Q S}\right)$, term by term. Using Lemma 4.9 we see that:

1) $\left|\boldsymbol{y}_{C}^{*} \boldsymbol{x}_{C}\right|=\left|\boldsymbol{y}_{C}^{*} K^{-1} K \boldsymbol{x}_{C}\right|=\left|\boldsymbol{y}_{B}^{*} \boldsymbol{x}_{B}\right|$;

2) $\left|\boldsymbol{y}_{C}^{*}\right|\left|C_{D}\right|\left|\boldsymbol{x}_{C}\right|=\left|\boldsymbol{y}_{B}^{*} K\right|\left|C_{D}\right|\left|K^{-1} \boldsymbol{x}_{B}\right|=\left|\boldsymbol{y}_{B}^{*}\right|\left|K C_{D} K^{-1}\right|\left|\boldsymbol{x}_{B}\right|=\left|\boldsymbol{y}_{B}^{*}\right|\left|B_{D}\right|\left|\boldsymbol{x}_{B}\right|$;

3) $\left|\boldsymbol{y}_{C}^{*}\right|\left|C_{L} \boldsymbol{x}_{C}\right|=\left|\boldsymbol{y}_{B}^{*} K\right|\left|C_{L} K^{-1} \boldsymbol{x}_{B}\right|=\left|\boldsymbol{y}_{B}^{*}\right|\left|K C_{L} K^{-1} \boldsymbol{x}_{B}\right|=\left|\boldsymbol{y}_{B}^{*}\right|\left|B_{L} \boldsymbol{x}_{B}\right|$;

4) $\left|\boldsymbol{y}_{C}^{*} C_{L}\right|\left|\boldsymbol{x}_{C}\right|=\left|\boldsymbol{y}_{B}^{*} K C_{L}\right|\left|K^{-1} \boldsymbol{x}_{B}\right|=\left|\boldsymbol{y}_{B}^{*} K C_{L} K^{-1}\right|\left|\boldsymbol{x}_{B}\right|=\left|\boldsymbol{y}_{B}^{*} B_{L}\right|\left|\boldsymbol{x}_{B}\right|$;

5) $\left|\boldsymbol{y}_{C}^{*}\right|\left|C_{U} \boldsymbol{x}_{C}\right|=\left|\boldsymbol{y}_{B}^{*} K\right|\left|C_{U} K^{-1} \boldsymbol{x}_{B}\right|=\left|\boldsymbol{y}_{B}^{*}\right|\left|K C_{U} K^{-1} \boldsymbol{x}_{B}\right|=\left|\boldsymbol{y}_{B}^{*}\right|\left|B_{U} \boldsymbol{x}_{B}\right|$;

6) $\left|\boldsymbol{y}_{C}^{*} C_{U}\right|\left|\boldsymbol{x}_{C}\right|=\left|\boldsymbol{y}_{B}^{*} K C_{U}\right|\left|K^{-1} \boldsymbol{x}_{B}\right|=\left|\boldsymbol{y}_{B}^{*} K C_{U} K^{-1}\right|\left|\boldsymbol{x}_{B}\right|=\left|\boldsymbol{y}_{B}^{*} B_{U}\right|\left|\boldsymbol{x}_{B}\right|$;

7) $\left|\boldsymbol{y}_{C}^{*}\left[\begin{array}{cc}0 & 0 \\ C(i+1: n, 1: i-1) & 0\end{array}\right] \boldsymbol{x}_{C}\right|=\left|\boldsymbol{y}_{B}^{*}\left[\begin{array}{cc}0 & 0 \\ B(i+1: n, 1: i-1) & 0\end{array}\right] \boldsymbol{x}_{B}\right|$;

8) $\left|\boldsymbol{y}_{C}^{*}\left[\begin{array}{cc}0 & C(1: j-1, j+1: n) \\ 0 & 0\end{array}\right] \boldsymbol{x}_{C}\right|=\left|\boldsymbol{y}_{B}^{*}\left[\begin{array}{cc}0 & C(1: j-1, j+1: n) \\ 0 & 0\end{array}\right] \boldsymbol{x}_{B}\right|$.

The result follows from Theorem 4.4, and from items 1) through 8) above. 


\subsection{Fast computation of the eigenvalue condition number}

The main contribution of this section is that, via Proposition 4.12, we will provide an algorithm for computing the eigenvalue condition number $\operatorname{cond}\left(\lambda ; \Omega_{Q S}\right)$ for any simple eigenvalue of any $\{1 ; 1\}$ quasiseparable matrix $C$ of size $n \times n$ in $\mathcal{O}(n)$ operations. Taking into account that fast algorithms for computing all the eigenvalues of a quasiseparable matrix cost $\mathcal{O}\left(n^{2}\right)$ flops [43], our result allows us to compute the condition numbers of all the eigenvalues of a quasiseparable matrix also in $\mathcal{O}\left(n^{2}\right)$ flops.

We remark that the difficulty of computing cond $\left(\lambda ; \Omega_{Q S}\right)$ fast comes mainly from the terms:

$$
\sum_{i=2}^{n-1}\left|\boldsymbol{y}^{*}\left[\begin{array}{cc}
0 & 0 \\
C(i+1: n, 1: i-1) & 0
\end{array}\right] \boldsymbol{x}\right| \text { and } \sum_{j=2}^{n-1} \mid \boldsymbol{y}^{*}\left[\begin{array}{cc}
0 & C(1: j-1, j+1: n) \\
0 & \boldsymbol{x}
\end{array} \mid\right.
$$

that appear in the formula given in Theorem 4.4. Note that the computation of these terms may be avoided, as a consequence of Proposition 4.8 , if we estimate cond $\left(\lambda ; \Omega_{Q S}\right)$ via $\operatorname{cond}_{\mathrm{eff}}\left(\lambda ; \Omega_{Q S}\right)$.

Proposition 4.12. Let $C \in \mathbb{R}^{n \times n}$ be a $\{1 ; 1\}$-quasiseparable matrix with a simple eigenvalue $\lambda \neq 0$ with left eigenvector $\boldsymbol{y}$ and right eigenvector $\boldsymbol{x}$, and assume that $\lambda, \boldsymbol{x}, \boldsymbol{y}$, and a quasiseparable representation $\Omega_{Q S}$ of $C$ are all known. Then, cond $\left(\lambda ; \Omega_{Q S}\right)$ can be computed in $42 n-66$ flops.

Proof. This proof consists of giving an algorithm for calculating cond $\left(\lambda ; \Omega_{Q S}\right)$. We will count the number of flops needed for calculating the expression of cond $\left(\lambda ; \Omega_{Q S}\right)$, term by term as follows.

(a) The factor $|\lambda|\left|\boldsymbol{y}^{*} \boldsymbol{x}\right|=\left|\lambda \sum_{i=1}^{n} \bar{y}_{i} x_{i}\right|$ requires $2 n$ flops.

(b) Since every product $\bar{y}_{i} x_{i}$ has already been calculated in (a), the term $\left|\boldsymbol{y}^{*}\right|\left|C_{D}\right||\boldsymbol{x}|=\sum_{i=1}^{n}\left|d_{i}\right|\left|\bar{y}_{i} x_{i}\right|$, can be calculated in $2 n-1$ flops.

(c) For the term $\left|\boldsymbol{y}^{*}\right|\left|C_{L} \boldsymbol{x}\right|$, we will calculate the products $C_{L} \boldsymbol{x}$ and $C_{L}^{(-2)} \boldsymbol{x}$ simultaneously, where $C_{L}^{(-2)}$ denotes the matrix that is obtained from $C_{L}$ by setting to zero the entries $\left(C_{L}\right)_{i+1, i}$ for $i=1,2, \ldots, n-1$, and that will be used later for calculating the term in (g). For simplicity, let us denote the vectors wlx $=C_{L}^{(-2)} \boldsymbol{x}$ and $\mathrm{zlx}=C_{L} \boldsymbol{x}$. Notice that wlx ${ }_{1}=\mathrm{wlx}_{2}=0$ and $\mathrm{zlx}_{1}=0$. The fast method for calculating $C_{L} \boldsymbol{x}$ is given by the following algorithm.

Routine 1 (Computes zlx $=C_{L} \boldsymbol{x}$ and wlx $=C_{L}^{(-2)} \boldsymbol{x}$ taking as inputs the quasiseparable parameters $\left\{p_{i}\right\}_{i=2}^{n},\left\{a_{i}\right\}_{i=2}^{n-1},\left\{q_{i}\right\}_{i=1}^{n-1}$, and the entries $\left\{x_{i}\right\}_{i=1}^{n}$ of $\boldsymbol{x}$.)

$\mathrm{zlx}_{1}=\mathrm{wlx}_{1}=\mathrm{wlx}_{2}=0$

$\mathrm{tlx} 1=q_{1} \cdot x_{1}$

$\mathrm{zlx}_{2}=p_{2} \cdot \mathrm{tlx} 1$

for $i=3: n$

$$
\begin{aligned}
& \mathrm{tlx} 2=a_{i-1} \cdot \mathrm{tlx} 1 \\
& \mathrm{wlx}_{i}=p_{i} \cdot \mathrm{tlx} 2 \\
& \mathrm{tlx} 1=\mathrm{tlx} 2+q_{i-1} \cdot x_{i-1} \\
& \operatorname{zlx}_{i}=p_{i} \cdot \mathrm{tlx} 1
\end{aligned}
$$

endf or

The fact that Routine 1 indeed computes zlx $=C_{L} \boldsymbol{x}$ and wlx $=C_{L}^{(-2)} \boldsymbol{x}$ can be proved easily by induction. Observe that Routine 1 uses the temporary variables tlx1 and tlx2, in addition to the entries of the vectors zlx and wlx. We warn the reader that these variables will be used also in Algorithm 1 for computing $\operatorname{cond}\left(\lambda ; \Omega_{Q S}\right)$ and are described in the table right before Algorithm 1. From Routine 1, we see that the cost of calculating $C_{L} \boldsymbol{x}$ and $C_{L}^{(-2)} \boldsymbol{x}$ is $5 n-8$ flops, and taking into account that $\left(C_{L} \boldsymbol{x}\right)_{1}=0$ it is straightforward that the cost of calculating $\left|\boldsymbol{y}^{*}\right|\left|C_{L} \boldsymbol{x}\right|$ and $\left|C_{L}^{(-2)} \boldsymbol{x}\right|$ simultaneously is $7 n-11$ flops.

(d) For the term $\left|\boldsymbol{y}^{*} C_{L}\right||\boldsymbol{x}|$, we can use a similar procedure to that in Routine 1 to compute zly $=\boldsymbol{y}^{*} C_{L}$ and wly $=\boldsymbol{y}^{*} C_{L}^{(-2)}$ by starting with the last components of these two row vectors. As before, this can be done at the cost of $5 n-8$ flops, and then, the cost of calculating $\left|\boldsymbol{y}^{*} C_{L}\right||\boldsymbol{x}|$ and $\boldsymbol{y}^{*} C_{L}^{(-2)}$ is $7 n-11$ flops. 
(e) For the term $\left|\boldsymbol{y}^{*}\right|\left|C_{U} \boldsymbol{x}\right|$, we can also calculate zux $=C_{U} \boldsymbol{x}$ and wux $=C_{U}^{(+2)} \boldsymbol{x}$ (where $C_{U}^{(+2)}$ denotes the matrix that is obtained from $C_{U}$ by setting to zero the entries $\left.\left(C_{U}\right)_{i, i+1}\right)$ by using an analogous process to that in Routine 1 . Note that calculating $C_{U} \boldsymbol{x}$ is similar to computing $\boldsymbol{y}^{*} C_{L}$ since $\left(\boldsymbol{y}^{*} C_{L}\right)^{*}=C_{L}^{T} \boldsymbol{y}$. Therefore, the cost of calculating $\left|\boldsymbol{y}^{*}\right|\left|C_{U} \boldsymbol{x}\right|$ and $C_{U}^{(+2)} \boldsymbol{x}$ is of $7 n-11 \mathrm{flops}$.

(f) The term $\left|\boldsymbol{y}^{*} C_{U}\right||\boldsymbol{x}|$, can also be computed simultaneously with wuy $=\boldsymbol{y}^{*} C_{U}^{(+2)}$ at a cost of $7 n-11$ flops, since the computation of zuy $=\boldsymbol{y}^{*} C_{U}$ is similar to the calculation of $C_{L} \boldsymbol{x}$, since $\left(\boldsymbol{y}^{*} C_{U}\right)^{*}=$ $C_{U}^{T} \boldsymbol{y}$.

(g) Denote $\alpha_{i}=\boldsymbol{y}^{*}\left[\begin{array}{cc}0 & 0 \\ C(i+1: n, 1: i-1) & 0\end{array}\right] \boldsymbol{x}$, and note that

$$
\alpha_{i}=\boldsymbol{y}^{*}\left[\begin{array}{cc}
0 & 0 \\
C(i+2: n, 1: i) & 0
\end{array}\right] \boldsymbol{x}+\boldsymbol{y}^{*}\left[\begin{array}{cc}
0 & 0 \\
C(i+1,1: i-1) & 0 \\
0 & 0
\end{array}\right] \boldsymbol{x}-\boldsymbol{y}^{*}\left[\begin{array}{ccc}
0 & 0 & 0 \\
0 & C(i+2: n, i) & 0
\end{array}\right] \boldsymbol{x} .
$$

We have obtained the following recursive equation,

$$
\alpha_{i}=\alpha_{i+1}+\bar{y}_{i+1}\left(C_{L}^{(-2)} \boldsymbol{x}\right)_{i+1}-\left(\boldsymbol{y}^{*} C_{L}^{(-2)}\right)_{i} x_{i}
$$

Recall that $C_{L}^{(-2)} \boldsymbol{x}$ and $\boldsymbol{y}^{*} C_{L}^{(-2)}$ were already calculated in (c) and (d), respectively. Then, the recurrence above is completed with the following fact:

$$
\alpha_{n-1}=\boldsymbol{y}^{*}\left[\begin{array}{cc}
0 & 0 \\
C(n, 1: n-2) & 0
\end{array}\right] \boldsymbol{x}=\bar{y}_{n}\left(C_{L}^{(-2)} \boldsymbol{x}\right)_{n} .
$$

Therefore, we can calculate the set $\left\{\alpha_{n-1}, \alpha_{n-2}, \cdots, \alpha_{2}\right\}$ in $4 n-11$ flops and the cost of calculating $\sum_{i=2}^{n-1}\left|\alpha_{i}\right|$, is $5 n-14$ flops.

(h) Analogously to the term above, the last sum in the formula for cond $\left(\lambda ; \Omega_{Q S}\right)$ can be computed in $5 n-14$ flops via a similar recurrence relation for $\beta_{j}=\boldsymbol{y}^{*}\left[\begin{array}{cc}0 & C(1: j-1, j+1: n) \\ 0 & 0\end{array}\right] \boldsymbol{x}:$

$$
\beta_{j}=\beta_{j-1}+\bar{y}_{j-1}\left(C_{U}^{(+2)} \boldsymbol{x}\right)_{j-1}-\left(\boldsymbol{y}^{*} C_{U}^{(+2)}\right)_{j} x_{j}, \text { and } \beta_{2}=\bar{y}_{1}\left(C_{U}^{(+2)} \boldsymbol{x}\right)_{1} \text {. }
$$

Finally, by summing all the costs in $(a)-(h)$, and considering the expression for cond $\left(\lambda ; \Omega_{Q S}\right)$, we conclude that the cost of computing it is $42 n-66$ flops.

\subsection{Pseudocode for computing cond $\left(\lambda ; \Omega_{Q S}\right)$ fast}

Based on the proof of Proposition 4.12 one can construct an algorithm for computing cond $\left(\lambda ; \Omega_{Q S}\right)$ fast. In this section we present, in Algorithm 1, the pseudocode for implementing such computations. If the reader is not interested in technical details, this section may be omitted. In the pseudocode we present, we will use the notation of the proof of Proposition 4.12. We will also use the functions 'zeros' (zeros $(m, n)$ returns an $m \times n$ matrix with zero entries), 'sum' $(\operatorname{sum}(\boldsymbol{z})$ returns the sum of the entries of the input vector $\boldsymbol{z}$ ) and 'conj' ( $\operatorname{conj}(\boldsymbol{z})$ returns an array of the same size of $\boldsymbol{z}$ such that its entries are the conjugates of the respective entries of $\boldsymbol{z}$ ) from Matlab. We denote by $\boldsymbol{d}$ the column vector of size $n$, such that $\boldsymbol{d}_{i}=d_{i}$. In the following table, we briefly describe the different variables that appear in Algorithm 1. 


\begin{tabular}{|l|l|}
\hline Variable & Description \\
\hline zlx & zlx $=C_{L} \boldsymbol{x}$ \\
\hline wlx & wlx $=C_{L}^{(-2)} \boldsymbol{x}$ \\
\hline zly & zly $=\boldsymbol{y}^{*} C_{L}$ \\
\hline wly & wly $=\boldsymbol{y}^{*} C_{L}^{(-2)}$ \\
\hline zux & zux $=C_{U} \boldsymbol{x}$ \\
\hline wux & wux $=C_{U}^{(+2)} \boldsymbol{x}$ \\
\hline zuy & zuy $=\boldsymbol{y}^{*} C_{U}$ \\
\hline wuy & wuy $=\boldsymbol{y}^{*} C_{U}^{(+2)}$ \\
\hline tlx1 & temporary variable introduced for the fast computation of zlx $=C_{L} \boldsymbol{x}$ \\
\hline tly1 & temporary variable introduced for the fast computation of zly $=\boldsymbol{y}^{*} C_{L}$ \\
\hline tux1 & temporary variable introduced for the fast computation of zux $=C_{U} \boldsymbol{x}$ \\
\hline tuy1 & temporary variable introduced for the fast computation of zuy $=\boldsymbol{y}^{*} C_{U}$ \\
\hline tlx2 & temporary variable introduced for the fast computation of wlx $=C_{L}^{(-2)} \boldsymbol{x}$ \\
\hline tly2 & temporary variable introduced for the fast computation of wly $=\boldsymbol{y}^{*} C_{L}^{(-2)}$ \\
\hline tux2 & temporary variable introduced for the fast computation of wux $=C_{U}^{(+2)} \boldsymbol{x}$ \\
\hline tuy2 & temporary variable introduced for the fast computation of wuy $=\boldsymbol{y}^{*} C_{U}^{(+2)}$ \\
\hline$\alpha$ & vector such that $\alpha(i)=\alpha_{i}$ with $\alpha_{i}$ as in $(\mathrm{g})$ in the proof of Proposition 4.12 \\
\hline$\beta$ & vector such that $\beta(i)=\beta_{i}$ with $\beta_{i}$ as in (h) in the proof of Proposition 4.12 \\
\hline
\end{tabular}

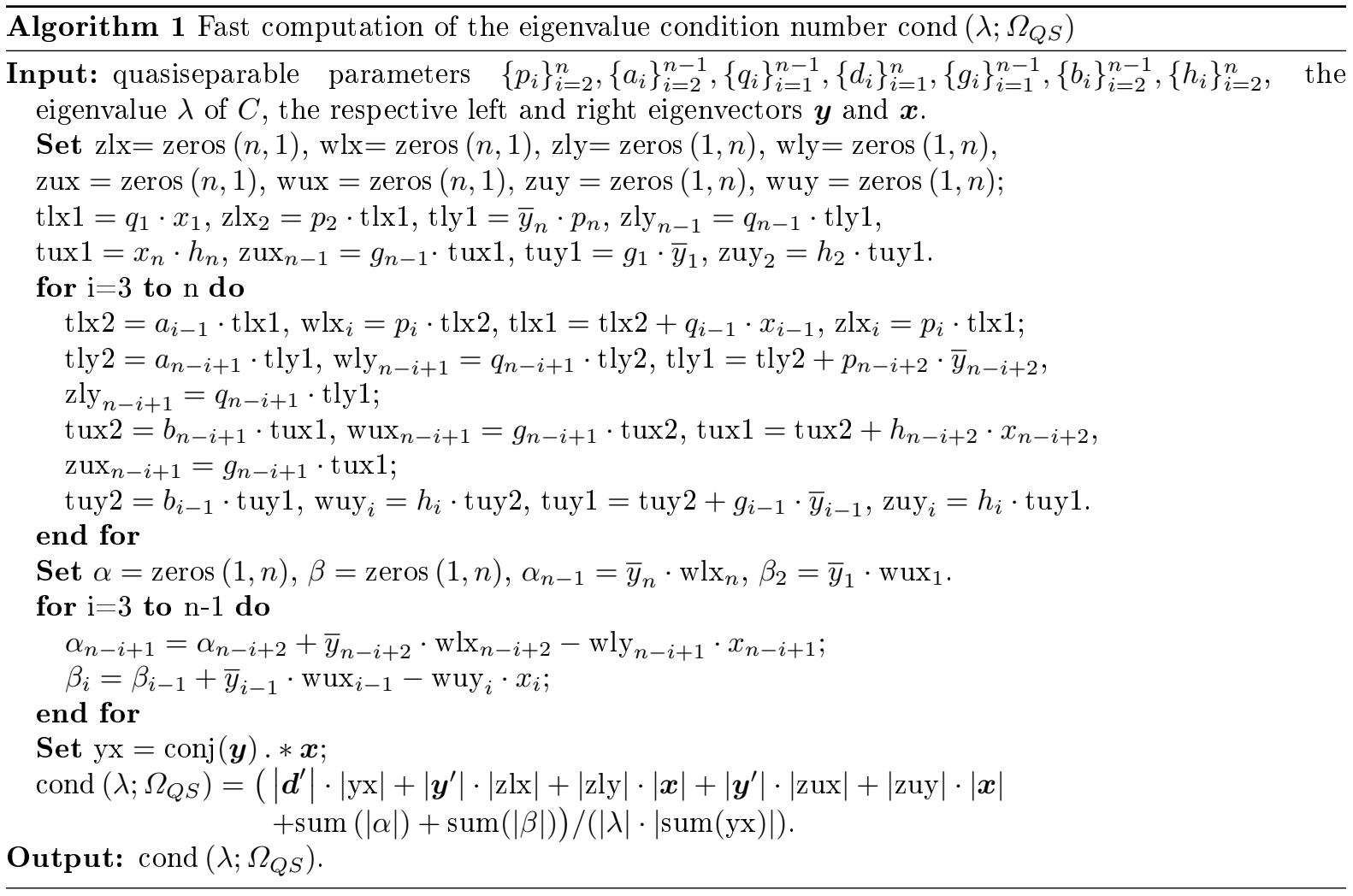

We remark that the temporary variables tlx1, tlx2, tly1, tly2, tux1, tux2, tuy1, and tuy2 have been introduced in order to save operations.

\section{Eigenvalue condition numbers for $\{1 ; 1\}$-quasiseparable matri- ces in the Givens-vector representation}

This section has, partially, a similar structure to the previous one. We will devote Section 5.1 to describe the Givens-vector representation [40], while the new results on eigenvalue condition numbers for this representation are presented in Sections 5.2, 5.3 and 5.4. However, some of the most important 
properties of these condition numbers will be studied in Section 6 .

\subsection{The Givens-vector representation for $\{1 ; 1\}$-quasiseparable matrices}

Another important representation for $\{1 ; 1\}$-quasiseparable matrices is the Givens-vector representation introduced in [40]. This representation was introduced to improve the numerical stability of fast matrix computations involving quasiseparable matrices with respect to other representations, but a rigorous proof that this is indeed the case has never been given. The results in this paper are a first contribution to the solution of this problem. The next theorem shows that this representation is able of representing the complete class of $\{1 ; 1\}$-quasiseparable matrices (see [42, Sections 2.4 and 2.8$]$ ).

Theorem 5.1. A matrix $C \in \mathbb{R}^{n \times n}$ is a $\{1 ; 1\}$-quasiseparable matrix if and only if it can be parameterized in terms of the following set of parameters,

- $\left\{c_{i}, s_{i}\right\}_{i=2}^{n-1}$, where $\left(c_{i}, s_{i}\right)$ is a pair of cosine-sine with $c_{i}^{2}+s_{i}^{2}=1$ for every $i \in\{2,3, \cdots, n-1\}$,

- $\left\{v_{i}\right\}_{i=1}^{n-1},\left\{d_{i}\right\}_{i=1}^{n},\left\{e_{i}\right\}_{i=1}^{n-1}$ all of them independent real parameters,

- $\left\{r_{i}, t_{i}\right\}_{i=2}^{n-1}$, where $\left(r_{i}, t_{i}\right)$ is a pair of cosine-sine with $r_{i}^{2}+t_{i}^{2}=1$ for every $i \in\{2,3, \cdots, n-1\}$, as follows:

$C=\left[\begin{array}{rrrrrr}d_{1} & e_{1} r_{2} & e_{1} t_{2} r_{3} & \cdots & e_{1} t_{2} \ldots t_{n-2} r_{n-1} & e_{1} t_{2} \ldots t_{n-1} \\ c_{2} v_{1} & d_{2} & e_{2} r_{3} & \cdots & e_{2} t_{3} \ldots t_{n-2} r_{n-1} & e_{2} t_{3} \ldots t_{n-1} \\ c_{3} s_{2} v_{1} & c_{3} v_{2} & d_{3} & \cdots & e_{3} t_{4} \ldots t_{n-2} r_{n-1} & e_{3} t_{4} \ldots t_{n-1} \\ \vdots & \vdots & \vdots & \ddots & \vdots & \vdots \\ c_{n-1} s_{n-2} \ldots s_{2} v_{1} & c_{n-1} s_{n-2} \ldots s_{3} v_{2} & c_{n-1} s_{n-2} \ldots s_{4} v_{3} & \cdots & d_{n-1} & e_{n-1} \\ s_{n-1} s_{n-2} \ldots s_{2} v_{1} & s_{n-1} s_{n-2} \ldots s_{3} v_{2} & s_{n-1} s_{n-2} \ldots s_{4} v_{3} & \cdots & v_{n-1} & d_{n}\end{array}\right]$.

This representation is denoted by $\Omega_{Q S}^{G V}$, i.e., $\Omega_{Q S}^{G V}:=\left(\left\{c_{i}, s_{i}\right\}_{i=2}^{n-1},\left\{v_{i}\right\}_{i=1}^{n-1},\left\{d_{i}\right\}_{i=1}^{n},\left\{e_{i}\right\}_{i=1}^{n-1},\left\{r_{i}, t_{i}\right\}_{i=2}^{n-1}\right)$.

From Theorems 4.1 and 5.1, it is obvious that the Givens-vector representation is a particular case of the quasiseparable representation for $\{1 ; 1\}$-quasiseparable matrices by considering the following relations between the parameters in Theorems 4.1 and 5.1, respectively: $\left\{p_{i}, a_{i}\right\}_{i=2}^{n-1}=\left\{c_{i}, s_{i}\right\}_{i=2}^{n-1},\left\{q_{i}\right\}_{i=1}^{n-1}=$ $\left\{v_{i}\right\}_{i=1}^{n-1},\left\{d_{i}\right\}_{i=1}^{n}=\left\{d_{i}\right\}_{i=1}^{n},\left\{g_{i}\right\}_{i=1}^{n-1}=\left\{e_{i}\right\}_{i=1}^{n-1},\left\{b_{i}, h_{i}\right\}_{i=2}^{n-1}=\left\{t_{i}, r_{i}\right\}_{i=2}^{n-1}$, and $p_{n}=h_{n}=1$. This fact can be observed better by comparing the expression in Example 4.2 and the expression in the following $5 \times 5$ example.

Example 5.2. Let $C \in \mathbb{R}^{5 \times 5}$ be a $\{1 ; 1\}$-quasiseparable matrix, and let

$$
\Omega_{Q S}^{G V}:=\left(\left\{c_{i}, s_{i}\right\}_{i=2}^{n-1},\left\{v_{i}\right\}_{i=1}^{n-1},\left\{d_{i}\right\}_{i=1}^{n},\left\{e_{i}\right\}_{i=1}^{n-1},\left\{r_{i}, t_{i}\right\}_{i=2}^{n-1}\right)
$$

be a Givens-vector representation of $C$. Then,

$$
C=\left[\begin{array}{rrrrr}
d_{1} & e_{1} r_{2} & e_{1} t_{2} r_{3} & e_{1} t_{2} t_{3} r_{4} & e_{1} t_{2} t_{3} t_{4} \\
c_{2} v_{1} & d_{2} & e_{2} r_{3} & e_{2} t_{3} r_{4} & e_{2} t_{3} t_{4} \\
c_{3} s_{2} v_{1} & c_{3} v_{2} & d_{3} & e_{3} r_{4} & e_{3} t_{4} \\
c_{4} s_{3} s_{2} v_{1} & c_{4} s_{3} v_{2} & c_{4} v_{3} & d_{4} & e_{4} \\
s_{4} s_{3} s_{2} v_{1} & s_{4} s_{3} v_{2} & s_{4} v_{3} & v_{4} & d_{5}
\end{array}\right] .
$$

Note that the Givens-vector representation can be made unique if $c_{i}$ and $r_{i}$ are taken to be nonnegative numbers (if $c_{i}=0$, take $s_{i}=1$ and if $r_{i}=0$, take $t_{i}=1$ ) [42, p.76].

\subsection{The eigenvalue condition number for $\{1 ; 1\}$-quasiseparable matrices in the Givens-vector representation}

Since the Givens-vector representation is a particular case of a quasiseparable representation, one might think that it makes no sense to study again eigenvalue condition numbers because we know, from Proposition 4.5, that they are independent of the particular choice of $\Omega_{Q S}$. However, the subtle point here is that the Givens-vector representation has correlated parameters since the pairs $\left\{c_{i}, s_{i}\right\}$ are not independent; the same happens for $\left\{r_{i}, t_{i}\right\}$. Since arbitrary componentwise perturbations of $\Omega_{Q S}^{G V}$ destroy the 
cosine-sine pairs, and we want to restrict ourselves to perturbations that preserve the cosine-sine pairs, an additional parametrization of these pairs is needed.

Avoiding the use of trigonometric functions, we essentially have two options for this additional parameters:

(a) We can consider $\left\{c_{i}, s_{i}\right\}=\left\{\sqrt{1-s_{i}^{2}}, s_{i}\right\}_{i=2}^{n-1}$ and $\left\{r_{i}, t_{i}\right\}=\left\{\sqrt{1-t_{i}^{2}}, t_{i}\right\}_{i=2}^{n-1}$, but this is not convenient because if $s_{i}$ is too close to 1 , then tiny relative variations of $s_{i}$ may produce large relative variations of $c_{i}$, since:

$$
\frac{s_{i}}{c_{i}} \frac{\partial c_{i}}{\partial s_{i}}=-\frac{s_{i}^{2}}{c_{i} \sqrt{1-s_{i}^{2}}}=-\left(\frac{s_{i}}{c_{i}}\right)^{2} .
$$

This is reflected in the following term of the eigenvalue condition number:

$$
\frac{s_{i}}{\lambda} \frac{\partial \lambda}{\partial s_{i}}=\frac{1}{\lambda\left(\boldsymbol{y}^{*} \boldsymbol{x}\right)} \boldsymbol{y}^{*}\left[\begin{array}{cc}
0 & 0 \\
-\left(\frac{s_{i}}{c_{i}}\right)^{2} C(i, 1: i-1) & 0 \\
C(i+1: n, 1: i-1) & 0
\end{array}\right] \boldsymbol{x}
$$

which may be huge if $\left(\frac{s_{i}}{c_{i}}\right)^{2}$ is huge. The same happens for $r_{i}$.

(b) On the other hand, we can use tangents as parameters in the following way:

$$
c_{i}=\frac{1}{\sqrt{1+l_{i}^{2}}}, s_{i}=\frac{l_{i}}{\sqrt{1+l_{i}^{2}}}, \text { and } r_{i}=\frac{1}{\sqrt{1+u_{i}^{2}}}, t_{i}=\frac{u_{i}}{\sqrt{1+u_{i}^{2}}}, \text { for } i=2, \ldots, n-1 .
$$

Observe that when using the tangents as parameters, the value $c_{i}=0$ (resp. $r_{i}=0$ ) corresponds to $l_{i}=\infty$ (resp. $\left.u_{i}=\infty\right)$. In addition, recall that, since we are interested in calculating relative-relative componentwise condition numbers, the parameters that are zero must remain zero. This is consistent with the fact that $\frac{\partial c_{i}}{\partial l_{i}}$ and $\frac{\partial s_{i}}{\partial l_{i}}$ both tend to zero when $l_{i} \rightarrow \infty$. The same happens with $\frac{\partial r_{i}}{\partial u_{i}}$ and $\frac{\partial t_{i}}{\partial u_{i}}$ when $u_{i} \rightarrow \infty$.

Since it is obvious from (b) that tiny relative perturbations of the tangents parameters $l_{i}$ and $u_{i}$ produce tiny relative perturbations of the cosine-sine parameters $\left\{c_{i}, s_{i}\right\}$ and $\left\{r_{i}, t_{i}\right\}$, respectively, it is convenient to use tangents parameters in practical numerical situations. This is related to and inspired by the fact that Givens rotations are computed in practice by using tangents or cotangents. See on this point the classical reference [26, Algorithm 5.1.3] and the state-of-the-art algorithm in [8].

Definition 5.3. For any Givens-vector representation

$$
\Omega_{Q S}^{G V}=\left(\left\{c_{i}, s_{i}\right\}_{i=2}^{n-1},\left\{v_{i}\right\}_{i=1}^{n-1},\left\{d_{i}\right\}_{i=1}^{n},\left\{e_{i}\right\}_{i=1}^{n-1},\left\{t_{i}, r_{i}\right\}_{i=2}^{n-1}\right)
$$

of a $\{1 ; 1\}$-quasiseparable matrix $C \in \mathbb{R}^{n \times n}$, we define the Givens-vector representation via tangents as

$$
\begin{gathered}
\Omega_{G V}:=\left(\left\{l_{i}\right\}_{i=2}^{n-1},\left\{v_{i}\right\}_{i=1}^{n-1},\left\{d_{i}\right\}_{i=1}^{n},\left\{e_{i}\right\}_{i=1}^{n-1},\left\{u_{i}\right\}_{i=2}^{n-1}\right), \text { where } \\
c_{i}=\frac{1}{\sqrt{1+l_{i}^{2}}}, s_{i}=\frac{l_{i}}{\sqrt{1+l_{i}^{2}}}, \text { and } r_{i}=\frac{1}{\sqrt{1+u_{i}^{2}}}, t_{i}=\frac{u_{i}}{\sqrt{1+u_{i}^{2}}}, \text { for } i=2, \ldots, n-1 .
\end{gathered}
$$

The next theorem is the main result of this section and it presents an explicit expression for calculating the componentwise eigenvalue condition number in the Givens-vector representation via tangents.

Theorem 5.4. Let $C \in \mathbb{R}^{n \times n}$ be a $\{1 ; 1\}$-quasiseparable matrix, let $\lambda \neq 0$ be a simple eigenvalue of $C$ with right eigenvector $\boldsymbol{x}$ and left eigenvector $\boldsymbol{y}$, and let $C=C_{L}+C_{D}+C_{U}$, with $C_{L}$ strictly lower triangular, $C_{D}$ diagonal, and $C_{U}$ strictly upper triangular. Then

$$
\begin{aligned}
\operatorname{cond}\left(\lambda ; \Omega_{G V}\right) & =\frac{1}{|\lambda|\left|\boldsymbol{y}^{*} \boldsymbol{x}\right|}\left\{\left|\boldsymbol{y}^{*}\right|\left|C_{D}\right||\boldsymbol{x}|+\left|\boldsymbol{y}^{*} C_{L}\right||\boldsymbol{x}|+\left|\boldsymbol{y}^{*}\right|\left|C_{U} \boldsymbol{x}\right|+\sum_{i=2}^{n-1}\left|\boldsymbol{y}^{*}\left[\begin{array}{cc}
0 \\
-s_{i}^{2} C(i, 1: i-1) & 0 \\
c_{i}^{2} C(i+1: n, 1: i-1) & 0
\end{array}\right] \boldsymbol{x}\right|\right. \\
& \left.+\sum_{j=2}^{n-1}\left|\boldsymbol{y}^{*}\left[\begin{array}{ccc}
0 & -t_{j}^{2} C(1: j-1, j) & r_{j}^{2} C(1: j-1, j+1: n) \\
0 & 0
\end{array}\right] \boldsymbol{x}\right|\right\} .
\end{aligned}
$$


Proof. As in the proof of Theorem 4.4, we will proceed term by term, using (2.5) in Theorem 2.13. Derivatives with respect to the parameters $\left\{l_{i}\right\}_{i=2}^{n-1}$ : Note first that

$$
\begin{gathered}
l_{i} \frac{\partial c_{i}}{\partial l_{i}}=-\frac{l_{i}^{2}}{\left(1+l_{i}^{2}\right)^{3 / 2}}=-s_{i}^{2} c_{i}, \quad l_{i} \frac{\partial s_{i}}{\partial l_{i}}=\frac{l_{i}}{\left(1+l_{i}^{2}\right)^{3 / 2}}=c_{i}^{2} s_{i}, \text { and, therefore, } \\
\quad \frac{l_{i}}{\lambda} \frac{\partial \lambda}{\partial l_{i}}=\frac{1}{\lambda\left(\boldsymbol{y}^{*} \boldsymbol{x}\right)}\left(\boldsymbol{y}^{*}\left[\begin{array}{cc}
0 & 0 \\
-s_{i}^{2} C(i, 1: i-1) & 0 \\
c_{i}^{2} C(i+1: n, 1: i-1) & 0
\end{array}\right] \boldsymbol{x}\right), i=2: n-1 .
\end{gathered}
$$

Derivatives with respect to the parameters $\left\{v_{j}\right\}_{j=1}^{n-1}$ : Note that

$$
\begin{gathered}
v_{j} \frac{\partial C}{\partial v_{j}}=\left[\begin{array}{ccccccc}
0 & \cdots & 0 & 0 & 0 & \cdots & 0 \\
0 & \cdots & 0 & C(j+1: n, j) & 0 & \cdots & 0
\end{array}\right], \text { which implies } \\
\frac{v_{j}}{\lambda} \frac{\partial \lambda}{\partial v_{j}}=\frac{1}{\lambda\left(\boldsymbol{y}^{*} \boldsymbol{x}\right)} \boldsymbol{y}^{*} C_{L}(:, j) x_{j} .
\end{gathered}
$$

Derivatives with respect to the parameters $\left\{d_{i}\right\}_{i=1}^{n}$ :

$$
\frac{d_{i}}{\lambda} \frac{\partial \lambda}{\partial d_{i}}=\frac{1}{\lambda\left(\boldsymbol{y}^{*} \boldsymbol{x}\right)} d_{i} \bar{y}_{i} x_{i} .
$$

Derivatives with respect to the parameters $\left\{e_{i}\right\}_{i=1}^{n-1}$ :

$$
\frac{e_{i}}{\lambda} \frac{\partial \lambda}{\partial e_{i}}=\frac{1}{\lambda\left(\boldsymbol{y}^{*} \boldsymbol{x}\right)} \bar{y}_{i} C_{U}(i,:) \boldsymbol{x} .
$$

Derivatives with respect to the parameters $\left\{u_{j}\right\}_{j=2}^{n-1}$ : We first note that:

$$
u_{j} \frac{\partial r_{j}}{\partial u_{j}}=-\frac{u_{j}^{2}}{\left(1+u_{j}^{2}\right)^{3 / 2}}=-t_{j}^{2} r_{j} \text { and } u_{j} \frac{\partial t_{j}}{\partial u_{j}}=\frac{u_{j}}{\left(1+u_{j}^{2}\right)^{3 / 2}}=r_{j}^{2} t_{j},
$$

from which we obtain that

$$
\frac{u_{j}}{\lambda} \frac{\partial \lambda}{\partial u_{j}}=\frac{1}{\lambda\left(\boldsymbol{y}^{*} \boldsymbol{x}\right)}\left(\boldsymbol{y}^{*}\left[\begin{array}{ccc}
0 & -t_{j}^{2} C(1: j-1, j) & r_{j}^{2} C(1: j-1, j+1: n) \\
0 & 0 & 0
\end{array}\right] \boldsymbol{x}\right) .
$$

Again, as in the proof of Theorem 4.4, we consider the sums involving the partial derivatives with respect to each subset of parameters:

$$
\begin{aligned}
& \text { - } k_{l}=\sum_{i=2}^{n-1}\left|\frac{l_{i}}{\lambda} \frac{\partial \lambda}{\partial l_{i}}\right|=\frac{1}{\left|\lambda\left(\boldsymbol{y}^{*} \boldsymbol{x}\right)\right|} \sum_{i=2}^{n-1}\left|\boldsymbol{y}^{*}\left[\begin{array}{cc}
-s_{i}^{2} C(i, 1: i-1) & 0 \\
c_{i}^{2} C(i+1: n, 1: i-1) & 0
\end{array}\right] \boldsymbol{x}\right| ; \\
& \text { - } k_{v}=\sum_{j=1}^{n-1}\left|\frac{v_{j}}{\lambda} \frac{\partial \lambda}{\partial v_{j}}\right|=\frac{1}{\left|\lambda\left(\boldsymbol{y}^{*} \boldsymbol{x}\right)\right|} \sum_{j=1}^{n-1}\left|\boldsymbol{y}^{*} C_{L}(:, j) x_{j}\right|=\frac{1}{\left|\lambda\left(\boldsymbol{y}^{*} \boldsymbol{x}\right)\right|}\left|\boldsymbol{y}^{*} C_{L}\right||\boldsymbol{x}| ; \\
& \text { - } k_{d}=\sum_{i=1}^{n}\left|\frac{d_{i}}{\lambda} \frac{\partial \lambda}{\partial d_{i}}\right|=\frac{1}{\left|\lambda\left(\boldsymbol{y}^{*} \boldsymbol{x}\right)\right|} \sum_{i=1}^{n}\left|d_{i} \bar{y}_{i} x_{i}\right|=\frac{1}{\left|\lambda\left(\boldsymbol{y}^{*} \boldsymbol{x}\right)\right|}\left|\boldsymbol{y}^{*}\right|\left|C_{D}\right||\boldsymbol{x}| ; \\
& \text { - } k_{e}=\sum_{i=1}^{n-1}\left|\frac{e_{i}}{\lambda} \frac{\partial \lambda}{\partial e_{i}}\right|=\frac{1}{\left|\lambda\left(\boldsymbol{y}^{*} \boldsymbol{x}\right)\right|} \sum_{i=1}^{n-1}\left|\bar{y}_{i} C_{U}(i,:) \boldsymbol{x}\right|=\frac{1}{\left|\lambda\left(\boldsymbol{y}^{*} \boldsymbol{x}\right)\right|}\left|\boldsymbol{y}^{*}\right|\left|C_{U} \boldsymbol{x}\right| ; \\
& \text { - } k_{u}=\sum_{j=2}^{n-1}\left|\frac{u_{j}}{\lambda} \frac{\partial \lambda}{\partial u_{j}}\right|=\frac{1}{\left|\lambda\left(\boldsymbol{y}^{*} \boldsymbol{x}\right)\right|} \sum_{j=2}^{n-1} \mid \boldsymbol{y}^{*}\left[\begin{array}{ccc}
0 & -t_{j}^{2} C(1: j-1, j) & \left.r_{j}^{2} C(1: j-1, j+1: n)\right] \boldsymbol{x} \mid \\
0 & 0
\end{array}\right] .
\end{aligned}
$$

From Theorem 2.13 we have $\operatorname{cond}\left(\lambda ; \Omega_{G V}\right)=k_{l}+k_{v}+k_{d}+k_{e}+k_{u}$. 
From the expression in Theorem 5.4 for the relative condition number in the Givens-vector representation for a given $\{1 ; 1\}$-quasiseparable matrix $C \in \mathbb{R}^{n \times n}$, we see that it does not only depend on the matrix entries, the eigenvalue $\lambda$ and on the eigenvectors $\boldsymbol{x}$ and $\boldsymbol{y}$, but it does also depend on the parameters $\left\{c_{i}, s_{i}\right\}$ and $\left\{r_{i}, t_{i}\right\}$, which are uniquely determined by the entries of $C$. Therefore, we have obtained an important difference with respect to the relative condition number in a quasiseparable representation presented in Theorem 4.4. Since the Givens-vector representation does not change trivially under diagonal similarities, because of these cosines-sines parameters, this condition number is not invariant under diagonal similarities as we can see from Example 5.5.

Example 5.5. Let $C \in \mathbb{R}^{3 \times 3}$ be the $\{1 ; 1\}$-quasiseparable matrix generated as in Theorem 5.1 by the set of Givens-vector parameters given by $\left\{c_{2}, s_{2}\right\}=\left\{2.3768 \times 10^{-1},-9.7134 \times 10^{-1}\right\},\left\{v_{1}, v_{2}\right\}=$ $\{9.8355,-2.9770\},\left\{d_{1}, d_{2}, d_{3}\right\}=\{11.437,-5.3162,9.7257\},\left\{e_{1}, e_{2}\right\}=\{1.7658,9.7074\},\left\{t_{2}, r_{2}\right\}=$ $\left\{-9.8216 \times 10^{-1}, 1.8806 \times 10^{-1}\right\}$, and denote by $\Omega_{G V}^{C}$ the respective tangent-Givens-vector representation. Consider the matrix $K=\operatorname{diag}(-1,-1,6)$, and denote by $\Omega_{G V}^{K C K^{-1}}$ the tangent-Givens-vector representation of the matrix $K C K^{-1}$. Then, for the simple eigenvalue $\lambda=14.120$ and the respective left and right eigenvectors $\boldsymbol{y}=\left[9.6472 \times 10^{-1}, 5.5889 \times 10^{-2},-2.5728 \times 10^{-1}\right]^{T}$ and $\boldsymbol{x}=\left[-4.7887 \times 10^{-1}, 3.4548 \times 10^{-1}, 8.0705 \times 10^{-1}\right]^{T}$ of $C$, we have:

$$
\operatorname{cond}\left(\lambda, C ; \Omega_{G V}^{C}\right)=1.1706 \neq \operatorname{cond}\left(\lambda, K C K^{-1} ; \Omega_{G V}^{K C K^{-1}}\right)=1.2485
$$

Nevertheless, we will prove in Proposition 6.4 that eigenvalue condition numbers with respect to the tangent-Givens-vector representation can not suffer large variations under diagonal similarities.

\subsection{Fast computation of the eigenvalue condition number}

In this section we prove that $\operatorname{cond}\left(\lambda ; \Omega_{G V}\right)$ can be computed fast. This fact is stated in Proposition 5.6, which will be proved by providing an algorithm for computing $\operatorname{cond}\left(\lambda ; \Omega_{G V}\right)$ in $\mathcal{O}(n)$ operations.

Proposition 5.6. Let $C \in \mathbb{R}^{n \times n}$ be a $\{1 ; 1\}$-quasiseparable matrix with a simple eigenvalue $\lambda \neq 0$ with left eigenvector $\boldsymbol{y}$ and right eigenvector $\boldsymbol{x}$, and assume that $\lambda, \boldsymbol{x}, \boldsymbol{y}$, and the Givens-vector representation via tangents $\Omega_{G V}$ of $C$ are all known. Then, cond $\left(\lambda ; \Omega_{G V}\right)$ can be computed in $60 n-106$ flops.

Proof. As we did in the proof of Proposition 4.12, we will find the cost of calculating each term in the expression for cond $\left(\lambda ; \Omega_{G V}\right)$.

(a) In the first place, note that the cost of calculating $c_{i}=\frac{1}{\sqrt{1+l_{i}^{2}}}$ is obviously 4 flops, and the cost of computing $s_{i}=\frac{l_{i}}{\sqrt{1+l_{i}^{2}}}$, is only 1 flop because we have already calculated the denominator $\sqrt{1+l_{i}^{2}}$. Since the same holds for $r_{i}$ and $t_{i}$, we conclude that the total cost of calculating $\left\{c_{i}, s_{i}\right\}_{i=2}^{n-1}$ and $\left\{r_{i}, t_{i}\right\}_{i=2}^{n-1}$ is $10 n-20$ flops.

(b) The total cost of computing $\left\{c_{i}^{2}\right\}_{i=2}^{n-1},\left\{s_{i}^{2}\right\}_{i=2}^{n-1},\left\{r_{i}^{2}\right\}_{i=2}^{n-1}$, and $\left\{t_{i}^{2}\right\}_{i=2}^{n-1}$ is $4 n-8$ flops.

(c) The factor $\left|\lambda \boldsymbol{y}^{*} \boldsymbol{x}\right|=\left|\lambda \sum_{i=1}^{n} \bar{y}_{i} x_{i}\right|$ can be calculated in $2 n$ flops.

(d) Since every product $\bar{y}_{i} x_{i}$ has already been calculated in (c), the term $\left|\boldsymbol{y}^{*}\right|\left|C_{D}\right||\boldsymbol{x}|=\sum_{i=1}^{n}\left|d_{i}\right|\left|\bar{y}_{i} x_{i}\right|$, can be calculated in $2 n-1$ flops.

(e) For computing the products $\boldsymbol{y}^{*} C_{L}, C_{U} \boldsymbol{x}$ (that explicitly appear in the expression for cond $\left(\lambda ; \Omega_{G V}\right)$ ) and $C_{L} \boldsymbol{x}, \boldsymbol{y}^{*} C_{U}$ (which will be, respectively, needed in (g) and (h) in this proof) we can proceed as in (d),(e),(c),(f) in the proof of Proposition 4.12, respectively. In these processes, we obtain simultaneously $\boldsymbol{y}^{*} C_{L}^{(-2)}, C_{U}^{(+2)} \boldsymbol{x}, C_{L}^{(-2)} \boldsymbol{x}$, and $\boldsymbol{y}^{*} C_{U}^{(+2)}$ (which will also be needed for the fast computation of the last two sums in cond $\left.\left(\lambda ; \Omega_{G V}\right)\right)$. The total cost of these computations is $20 n-32$ flops.

(f) The products $\left|\boldsymbol{y}^{*} C_{L}\right||\boldsymbol{x}|$ and $\left|\boldsymbol{y}^{*}\right|\left|C_{U} \boldsymbol{x}\right|$, can be calculated at a cost of $2 n-3$ flops each. 
(g) Denote $\widetilde{\alpha}_{i}:=\boldsymbol{y}^{*}\left[\begin{array}{cc}0 & 0 \\ -s_{i}^{2} C(i, 1: i-1) & 0 \\ c_{i}^{2} C(i+1: n, 1: i-1) & 0\end{array}\right] \boldsymbol{x}$, and note that

$$
\begin{aligned}
\widetilde{\alpha}_{i} & =\boldsymbol{y}^{*}\left[\begin{array}{cc}
0 & 0 \\
-s_{i}^{2} C(i, 1: i-1) & 0 \\
0 & 0
\end{array}\right] \boldsymbol{x}+\boldsymbol{y}^{*}\left[\begin{array}{cc}
0 & 0 \\
0 & 0 \\
c_{i}^{2} C(i+1: n, 1: i-1) & 0
\end{array}\right] \boldsymbol{x} \\
& =-s_{i}^{2} \bar{y}_{i}\left(C_{L} \boldsymbol{x}\right)_{i}+c_{i}^{2}\left(\boldsymbol{y}^{*}\left[\begin{array}{cc}
0 & 0 \\
C(i+1: n, 1: i-1) & 0
\end{array}\right] \boldsymbol{x}\right) .
\end{aligned}
$$

The expression $-s_{i}^{2} \bar{y}_{i}\left(C_{L} \boldsymbol{x}\right)_{i}$ can be calculated in 2 flops since $s_{i}^{2}$ and $C_{L} \boldsymbol{x}$ have been calculated already. On the other hand, all the expressions $\left(\boldsymbol{y}^{*}\left[\begin{array}{cc}0 & 0 \\ C(i+1: n, 1: i-1) & 0\end{array}\right] \boldsymbol{x}\right)$ can be calculated via a recurrence relation as in $(\mathrm{g})$ in the proof of Proposition 4.12, in $4 n-11$ flops. Consequently, the vector $\left\{\widetilde{\alpha}_{i}\right\}_{i=2}^{n-1}$ can be calculated in $8 n-19$ flops, and the cost of computing $\sum_{i=2}^{n-1}\left|\widetilde{\alpha}_{i}\right|$ is $9 n-22$ flops.

(h) If we denote $\widetilde{\beta}_{j}:=\boldsymbol{y}^{*}\left[\begin{array}{ccc}0 & -t_{j}^{2} C(1: j-1, j) & r_{j}^{2} C(1: j-1, j+1: n) \\ 0 & 0 & 0\end{array}\right] \boldsymbol{x}$, then we can proceed in an analogous way to $(\mathrm{g})$, and obtain also a cost for computing $\sum_{j=2}^{n-1}\left|\widetilde{\beta}_{j}\right|$ of $9 n-22$ flops.

Finally, by summing all the costs obtained above, and from the expression for $\operatorname{cond}\left(\lambda ; \Omega_{G V}\right)$ we obtain a total cost of $60 n-106$ flops for computing $\operatorname{cond}\left(\lambda ; \Omega_{G V}\right)$.

\subsection{Pseudocode for computing cond $\left(\lambda ; \Omega_{G V}\right)$ fast}

In this section we present the pseudocode in Algorithm 2 for computing cond $\left(\lambda ; \Omega_{G V}\right)$ fast. Again, if the reader is not interested in such technical details then this section may be omitted.

Since any set of Givens-vector parameters can also be considered as a set of quasiseparable parameters, and since from the proof of Proposition 5.6 we know that cond $\left(\lambda ; \Omega_{G V}\right)$ can be calculated in a very similar way to cond $\left(\lambda ; \Omega_{Q S}\right)$, we have that Algorithms 1 and 2 are also similar. Therefore we do not describe Algorithm 2 in detail. We will use the standard functions 'zeros', 'sum', 'conj', and 'ones' from MATLAB. The vectors $\boldsymbol{d}$, zlx, wlx, zly, wly, zux, wux, zuy, wuy, $\alpha, \beta$, and the temporary variables tlx1, tly1, tux1, tuy1, tlx2, tly2, tux2, tuy2, are all defined as in Algorithm 1. In the following table, we briefly describe the new variables that appear in the pseudocode of Algorithm 2.

\begin{tabular}{|l|l|}
\hline Variable & Description \\
\hline$\widetilde{\alpha}$ & vector such that $\widetilde{\alpha}(i)=\widetilde{\alpha}_{i}$ with $\widetilde{\alpha}_{i}$ as in the proof of Proposition 5.6 \\
\hline$\widetilde{\beta}$ & vector such that $\widetilde{\beta}(i)=\widetilde{\beta}_{i}$ with $\widetilde{\beta}_{i}$ as in the proof of Proposition 5.6 \\
\hline
\end{tabular}




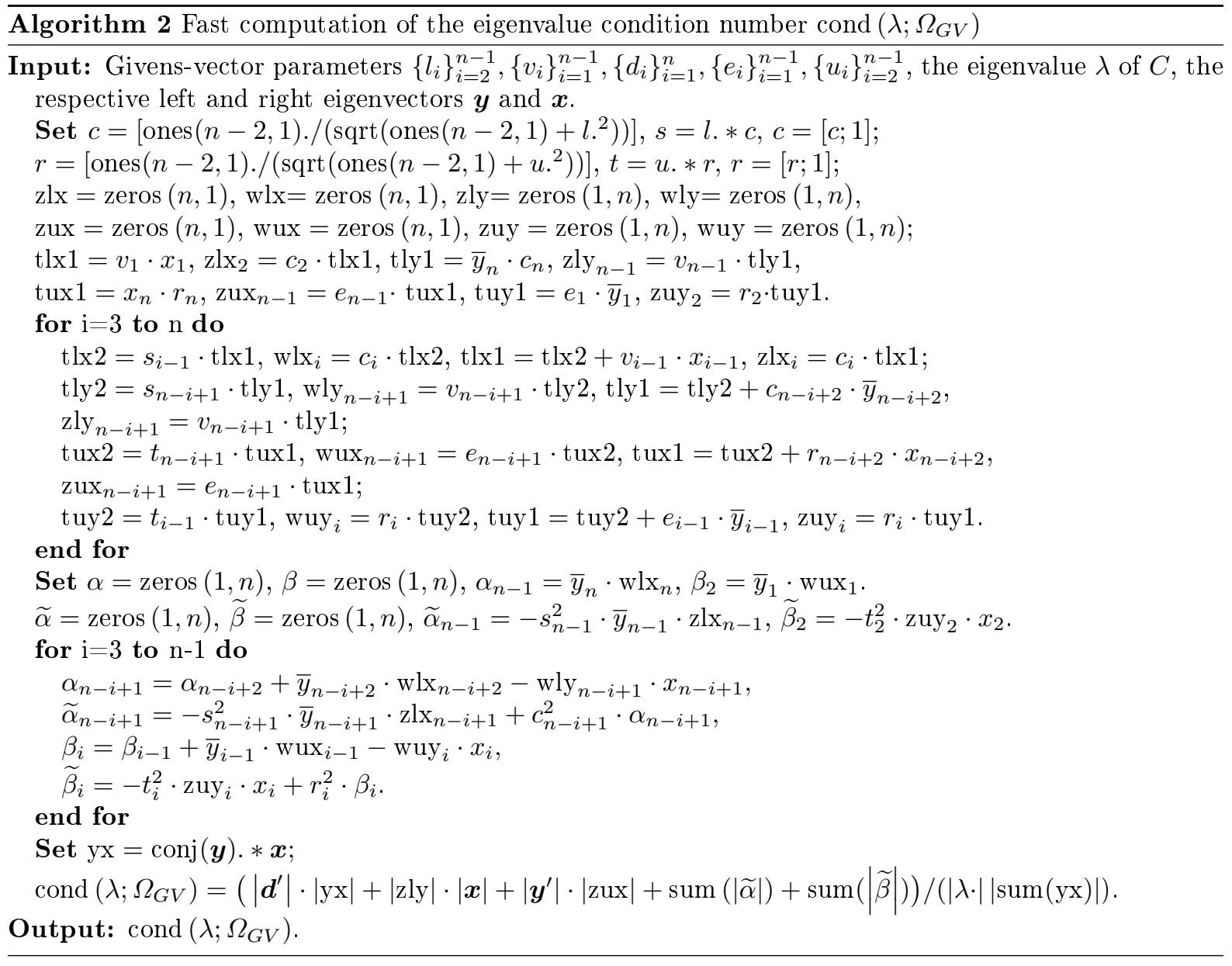

\section{Comparison of $\operatorname{cond}\left(\lambda ; \Omega_{Q S}\right)$ and $\operatorname{cond}\left(\lambda ; \Omega_{G V}\right)$}

As we know, the Givens-vector representation is a particular case of the quasiseparable representation which imposes additional constraints on the parameters. Since we will only consider perturbations respecting such constraints, that is, preserving the cosine-sine relations in the parameters $\left\{c_{i}, s_{i}\right\}$ and $\left\{r_{i}, t_{i}\right\}$ of $\Omega_{Q S}^{G V}$, it is natural to expect $\operatorname{cond}\left(\lambda ; \Omega_{G V}\right)$ not to be larger than cond $\left(\lambda ; \Omega_{Q S}\right)$. In Theorem 6.1, we prove that the Givens-vector representation via tangents is a "more stable" representation than the quasiseparable representation for eigenvalue computations for $\{1 ; 1\}$-quasiseparable matrices. This is the first time that a rigorous proof is given in such direction.

Theorem 6.1. Let $C \in \mathbb{R}^{n \times n}$ be a $\{1 ; 1\}$-quasiseparable matrix, $\Omega_{G V}$ be the tangent-Givens-vector parameters of $C, \Omega_{Q S}$ be any set of quasiseparable parameters of $C$, and $\lambda \neq 0$ be a simple eigenvalue of C. Then,

$$
\operatorname{cond}\left(\lambda ; \Omega_{G V}\right) \leq \operatorname{cond}\left(\lambda ; \Omega_{Q S}\right) .
$$

Proof. We compare for the same matrix $C$, the expression given for $\operatorname{cond}\left(\lambda ; \Omega_{Q S}\right)$ in Theorem 4.4 and the expression given in Theorem 5.4 for $\operatorname{cond}\left(\lambda ; \Omega_{G V}\right)$. Starting from the sums in the last two terms of the expression for $\operatorname{cond}\left(\lambda ; \Omega_{G V}\right)$, we have:

$$
\begin{aligned}
S_{1} & =\sum_{i=2}^{n-1}\left|\boldsymbol{y}^{*}\left[\begin{array}{cc}
-s_{i}^{2} C(i, 1: i-1) & 0 \\
c_{i}^{2} C(i+1: n, 1: i-1) & 0
\end{array}\right] \boldsymbol{x}\right| \\
& \leq \sum_{i=2}^{n-1}\left|\bar{y}_{i} C_{L}(i,:) \boldsymbol{x}\right|+\sum_{i=2}^{n-1}\left|\boldsymbol{y}^{*}\left[\begin{array}{cc}
0 & 0 \\
C(i+1: n, 1: i-1) & 0
\end{array}\right] \boldsymbol{x}\right| \\
& =\left|\boldsymbol{y}^{*}\right|\left|C_{L} \boldsymbol{x}\right|+\sum_{i=2}^{n-1}\left|\boldsymbol{y}^{*}\left[\begin{array}{cc}
0 & 0 \\
C(i+1: n, 1: i-1) & 0
\end{array}\right] \boldsymbol{x}\right| .
\end{aligned}
$$


Analogously, we obtain:

$$
\begin{aligned}
S_{2} & =\sum_{j=2}^{n-1}\left|\boldsymbol{y}^{*}\left[\begin{array}{ccc}
0 & -t_{j}^{2} C(1: j-1, j) & r_{j}^{2} C(1: j-1, j+1: n) \\
0 & 0 & 0
\end{array}\right] \boldsymbol{x}\right| \\
& \leq\left|\boldsymbol{y}^{*} C_{U}\right||\boldsymbol{x}|+\sum_{j=2}^{n-1}\left|\boldsymbol{y}^{*}\left[\begin{array}{cc}
0 & C(1: j-1, j+1: n) \\
0 & 0
\end{array}\right] \boldsymbol{x}\right| .
\end{aligned}
$$

From (6.1) and (6.2) we have

$$
\begin{aligned}
\operatorname{cond}\left(\lambda ; \Omega_{G V}\right) & \leq \frac{1}{|\lambda|\left|\boldsymbol{y}^{*} \boldsymbol{x}\right|}\left\{\left|\boldsymbol{y}^{*}\right|\left|C_{D}\right||\boldsymbol{x}|+\left|\boldsymbol{y}^{*}\right|\left|C_{L} \boldsymbol{x}\right|+\left|\boldsymbol{y}^{*} C_{L}\right||\boldsymbol{x}|+\left|\boldsymbol{y}^{*}\right|\left|C_{U} \boldsymbol{x}\right|+\left|\boldsymbol{y}^{*} C_{U}\right||\boldsymbol{x}|\right. \\
& \left.+\sum_{i=2}^{n-1}\left|\boldsymbol{y}^{*}\left[\begin{array}{cc}
0 & 0 \\
C(i+1: n, 1: i-1) & 0
\end{array}\right] \boldsymbol{x}\right|+\sum_{j=2}^{n-1} \mid \boldsymbol{y}^{*}\left[\begin{array}{cc}
0 & C(1: j-1, j+1: n) \\
0 & 0
\end{array}\right]\right\} \\
& =\operatorname{cond}\left(\lambda ; \Omega_{Q S}\right) .
\end{aligned}
$$

On the other hand, we are going to show now that the Givens-vector representation via tangents can only improve, with respect to a general quasiseparable representation, the relative condition number of a simple eigenvalue of a quasiseparable matrix, up to a factor of $3 n$. Therefore, both representations can be considered equivalent from the point of view of the accuracy of the eigenvalue computations that they allow. This is proved in Theorem 6.3, for which we need the simple Lemma 6.2. It is worth to observe that results in the spirit of Lemma 6.2 can be found in the detailed error analysis of Givens rotations presented in [4].

Lemma 6.2. Let $l \neq 0$ be a real number representing a tangent and $c$ the corresponding positive cosine. Then, for any positive value $\eta<1$, a relative perturbation of $l$ by at most $\eta$ produces a relative perturbation of $c$ of the order of $\eta$, i.e.,

$$
\left|\frac{\delta l}{l}\right| \leq \eta \Longrightarrow\left|\frac{\delta c}{c}\right| \leq\left(\eta+\mathcal{O}\left(\eta^{2}\right)\right), \text { where } c+\delta c=\frac{1}{\sqrt{1+(l+\delta l)^{2}}} .
$$

Proof. Consider $l^{\prime}=l+\delta l$ as the perturbed tangent and $c^{\prime}=c+\delta c=1 / \sqrt{1+\left(l^{\prime}\right)^{2}}$ as the respective perturbed cosine. Then, for $1>\eta>0$ sufficiently small we have that if $(1-\eta)|l| \leq\left|l^{\prime}\right| \leq(1+\eta)|l|$, then

$$
\frac{c}{1+\eta}=\frac{1}{(1+\eta)\left(\sqrt{1+l^{2}}\right)} \leq c^{\prime} \leq \frac{1}{(1-\eta)\left(\sqrt{1+l^{2}}\right)}=\frac{c}{1-\eta},
$$

from where we can conclude that $|\delta l| \leq \eta|l| \Rightarrow|\delta c| \leq\left(\eta+\mathcal{O}\left(\eta^{2}\right)\right)|c|$.

Theorem 6.3. Let $\lambda \neq 0$ be a simple eigenvalue of a $\{1 ; 1\}$-quasiseparable matrix $C \in \mathbb{R}^{n \times n}$ and $\Omega_{G V}$ be the tangent-Givens-vector representation of $C$. Then for any quasiseparable representation $\Omega_{Q S}$ of $C$ :

$$
\frac{\operatorname{cond}\left(\lambda ; \Omega_{Q S}\right)}{\operatorname{cond}\left(\lambda ; \Omega_{G V}\right)} \leq 3(n-2) .
$$

Proof. Recall that from the Givens-vector representation via tangents $\Omega_{G V}$ of $C$ we can obtain the Givens-vector representation $\Omega_{Q S}^{G V}$ of $C$ as in Definition 5.3, and that $\Omega_{Q S}^{G V}$ is also a quasiseparable representation of $C$ as we explained after Theorem 5.1. Therefore, in order to use the definition of the componentwise relative eigenvalue condition number for representations, i.e., Definition 2.10, let us consider a quasiseparable perturbation $\delta \Omega_{Q S}^{G V}$ of the parameters in $\Omega_{Q S}^{G V}$ such that $\left|\delta \Omega_{Q S}^{G V}\right| \leq \eta\left|\Omega_{Q S}^{G V}\right|$, and the resulting quasiseparable matrix $\tilde{C}:=C\left(\Omega_{Q S}^{G V}+\delta \Omega_{Q S}^{G V}\right)$. We will refer to $\eta$ as the level of the relative perturbation of the parameters in the representation $\Omega_{Q S}^{G V}$. We emphasize that the perturbation $\delta \Omega_{Q S}^{G V}$ does not respect in general the pairs cosine-sine.

On the other hand, note that $\tilde{C}$ can also be represented by a set

$$
\Omega_{G V}^{\prime}:=\left(\left\{l_{i}^{\prime}\right\}_{i=2}^{n-1},\left\{v_{i}^{\prime}\right\}_{i=1}^{n-1},\left\{d_{i}^{\prime}\right\}_{i=1}^{n},\left\{e_{i}^{\prime}\right\}_{i=1}^{n-1},\left\{u_{i}^{\prime}\right\}_{i=2}^{n-1}\right)
$$


of tangent-Givens-vector parameters and let us consider the perturbations $\delta^{\prime} \Omega_{G V}:=\Omega_{G V}^{\prime}-\Omega_{G V}$. For simplicity, we will denote $\tilde{C}:=C\left(\Omega_{Q S}^{G V}+\delta \Omega_{Q S}^{G V}\right)=C\left(\Omega_{G V}^{\prime}\right)$. The strategy of the proof is to find an upper bound for the level $\eta^{\prime}$ of the respective relative perturbations in the parameters in $\Omega_{G V}^{\prime}$ produced by the level $\eta$ of relative perturbation in the quasiseparable parameters in $\Omega_{Q S}^{G V}$, i.e., we will find a function $\eta^{\prime}(\eta)$ such that $\left|\delta \Omega_{Q S}^{G V}\right| \leq \eta\left|\Omega_{Q S}^{G V}\right| \Rightarrow\left|\delta^{\prime} \Omega_{G V}\right| \leq \eta^{\prime}(\eta)\left|\Omega_{G V}\right|$. Let us proceed by analyzing each subset of parameters as follows.

- For the parameters in $\left\{d_{i}^{\prime}\right\}_{i=1}^{n}$ it is obvious that $\delta^{\prime} d_{i}=\delta d_{i}$.

- For the parameters in $\left\{v_{j}^{\prime}\right\}_{j=1}^{n-1}$ note that $v_{j}^{\prime}=\sqrt{\sum_{i=j+1}^{n}(\tilde{C}(i, j))^{2}}$ from where it is easy to see that if $\left|\delta \Omega_{Q S}^{G V}\right| \leq \eta\left|\Omega_{Q S}^{G V}\right|$, then

$$
\begin{aligned}
\sqrt{\sum_{i=j+1}^{n}\left[(1-\eta)^{n} C(i, j)\right]^{2}} & \leq v_{j}^{\prime} \leq \sqrt{\sum_{i=j+1}^{n}\left[(1+\eta)^{n} C(i, j)\right]^{2}}, \\
(1-\eta)^{n} \sqrt{\sum_{i=j+1}^{n}[C(i, j)]^{2}} & \leq v_{j}^{\prime} \leq(1+\eta)^{n} \sqrt{\sum_{i=j+1}^{n}[C(i, j)]^{2}}, \\
(1-\eta)^{n} v_{j} & \leq v_{j}^{\prime} \leq(1+\eta)^{n} v_{j},
\end{aligned}
$$

and, consequently, we have that $\left|\delta^{\prime} v_{j}\right| \leq\left(n \eta+\mathcal{O}\left(\eta^{2}\right)\right)\left|v_{j}\right|$, for $j=1, \ldots, n-1$.

- For studying the level of perturbation in the parameters $\left\{l_{i}^{\prime}\right\}_{i=2}^{n-1}$ produced by the level $\eta$ of perturbation in $\Omega_{Q S}^{G V}+\delta \Omega_{Q S}^{G V}$, recall first that the parameter $p_{n}=1$ of $\Omega_{Q S}^{G V}$ is also perturbed. Next, taking into account which are the entries of $\tilde{C}$ in the representations $\Omega_{Q S}^{G V}+\delta \Omega_{Q S}^{G V}$ and $\Omega_{G V}^{\prime}$ according to Theorems 4.1 and 5.1, observe

$$
\begin{aligned}
l_{n-1}^{\prime} & =\frac{s_{n-1}^{\prime}}{c_{n-1}^{\prime}}=\frac{\tilde{C}(n, n-2)}{\tilde{C}(n-1, n-2)}=\frac{\left(1+\epsilon_{n-1}\right) s_{n-1}\left(1+\alpha_{n-1}\right)}{c_{n-1}\left(1+\beta_{n-1}\right)}, \text { and } \\
l_{i}^{\prime} & =\frac{s_{i}^{\prime}}{c_{i}^{\prime}}=\frac{\tilde{C}(i+1, i-1)}{\tilde{C}(i, i-1)} \frac{1}{c_{i+1}^{\prime}}=\frac{c_{i+1}\left(1+\epsilon_{i}\right) s_{i}\left(1+\alpha_{i}\right)}{c_{i}\left(1+\beta_{i}\right) c_{i+1}^{\prime}}, \text { for } i=n-2, n-3, \ldots, 2,
\end{aligned}
$$

where $\left|\epsilon_{i}\right| \leq \eta,\left|\alpha_{i}\right| \leq \eta,\left|\beta_{i}\right| \leq \eta$, for $i=n-1, n-2, \ldots, 2$. From Lemma 6.2 , we know that if $\left|\delta^{\prime} l_{i+1}\right|=\left|l_{i+1}^{\prime}-l_{i+1}\right| \leq \kappa_{i+1}\left|l_{i+1}\right|$, then $\left|\delta^{\prime} c_{i+1}\right|=\left|c_{i+1}^{\prime}-c_{i+1}\right| \leq\left(\kappa_{i+1}+\mathcal{O}\left(\kappa_{i+1}^{2}\right)\right)\left|c_{i+1}\right|$, or equivalently $c_{i+1}^{\prime}=c_{i+1}\left(1+\theta_{i+1}\right)$, with $\left|\theta_{i+1}\right| \leq \kappa_{i+1}+\mathcal{O}\left(\kappa_{i+1}^{2}\right)$. Therefore, the second equation in (6.3) implies

$$
\left|\delta^{\prime} l_{i}\right|=\left|l_{i}^{\prime}-l_{i}\right| \leq\left(3 \eta+\kappa_{i+1}+\mathcal{O}\left(\eta^{2}+\kappa_{i+1}^{2}\right)\right)\left|l_{i}\right| \text { for } i=n-2, n-3, \ldots, 2,
$$

and the first equation in (6.3) implies $\left|\delta^{\prime} l_{n-1}\right| \leq\left(3 \eta+\mathcal{O}\left(\eta^{2}\right)\right)\left|l_{n-1}\right|$, i.e., up to first order in $\eta$, we can take $\kappa_{n-1}=3 \eta$. So, equation (6.4) provides a recurrence relation for $\kappa_{i+1}$ and we get

$$
\left|\delta^{\prime} l_{i}\right| \leq\left(3(n-i) \eta+\mathcal{O}\left(\eta^{2}\right)\right)\left|l_{i}\right| .
$$

- For the parameters in $\left\{e_{i}^{\prime}\right\}_{i=1}^{n-1}$, we can proceed in an analogous way to that for the parameters in $\left\{v_{j}^{\prime}\right\}_{j=1}^{n-1}$, and we obtain that $\left|\delta^{\prime} e_{i}\right| \leq\left(n \eta+\mathcal{O}\left(\eta^{2}\right)\right)\left|e_{i}\right|$, for $i=1, \ldots, n-1$.

- For the parameters in $\left\{u_{j}^{\prime}\right\}_{j=2}^{n-1}$, we can also proceed in an analogous way to that for the parameters in $\left\{l_{i}^{\prime}\right\}_{i=2}^{n-1}$, and we obtain that $\left|\delta^{\prime} u_{j}\right| \leq\left(3(n-j) \eta+\mathcal{O}\left(\eta^{2}\right)\right)\left|u_{j}\right|$, for $j=2, \ldots, n-1$.

Note that for a $\{1 ; 1\}$-quasiseparable matrix $C:=C\left(\Omega_{G V}\right)=C\left(\Omega_{Q S}^{G V}\right)$, we have proved, so far, that given any quasiseparable perturbation $\left|\delta \Omega_{Q S}^{G V}\right| \leq \eta\left|\Omega_{Q S}^{G V}\right|$, there exists a perturbation $\delta^{\prime} \Omega_{G V}$ of the tangent-Givens-vector parameters of $C$, such that $C\left(\Omega_{Q S}^{G V}+\delta \Omega_{Q S}^{G V}\right)=C\left(\Omega_{G V}+\delta^{\prime} \Omega_{G V}\right)$ and $\left|\delta^{\prime} \Omega_{G V}\right| \leq$ $\left(3(n-2) \eta+\mathcal{O}\left(\eta^{2}\right)\right)\left|\Omega_{G V}\right|$. Then, from Definition 2.10, we have:

$$
\begin{gathered}
\operatorname{cond}\left(\lambda ; \Omega_{Q S}\right) \leq \lim _{\eta \rightarrow 0} \sup \left\{\frac{|\delta \lambda|}{\eta|\lambda|}:(\lambda+\delta \lambda) \text { is an eigenvalue of } C\left(\Omega_{G V}+\delta \Omega_{G V}\right),\right. \\
\left.\left|\delta \Omega_{G V}\right| \leq\left(3(n-2) \eta+\mathcal{O}\left(\eta^{2}\right)\right)\left|\Omega_{G V}\right|\right\} .
\end{gathered}
$$


By considering the change of variable $\eta^{\prime}=\left(3(n-2) \eta+\mathcal{O}\left(\eta^{2}\right)\right)$, we obtain

$$
\begin{gathered}
\operatorname{cond}\left(\lambda ; \Omega_{Q S}\right) \leq \lim _{\eta^{\prime} \rightarrow 0} \sup \left\{\frac{3(n-2)|\delta \lambda|}{\eta^{\prime}|\lambda|}:(\lambda+\delta \lambda) \text { is an eigenvalue of } C\left(\Omega_{G V}+\delta \Omega_{G V}\right),\right. \\
\left.\left|\delta \Omega_{G V}\right| \leq \eta^{\prime}\left|\Omega_{G V}\right|\right\}=3(n-2) \operatorname{cond}\left(\lambda ; \Omega_{G V}\right) .
\end{gathered}
$$

From Example 5.5 in Section 5.2 we know that the eigenvalue condition number with respect to the tangent-Givens-vector representation is not invariant under diagonal similarities. However, from Theorems 6.1, 6.3, and 4.11, it is straightforward to prove that the variations that may be obtained in the condition number under these similarities are not significant from a numerical point of view. This is stated, without proof, in the following proposition.

Proposition 6.4. Let $\lambda \neq 0$ be a simple eigenvalue of a $\{1 ; 1\}$-quasiseparable matrix $C \in \mathbb{R}^{n \times n}, \Omega_{G V}$ be the tangent-Givens-vector representation of $C, K \in \mathbb{R}^{n \times n}$ be diagonal and nonsingular and $\Omega_{G V}^{K C K^{-1}}$ be the tangent-Givens-vector representation of $\mathrm{KCK}^{-1}$. Then

$$
\frac{1}{3(n-2)} \leq \frac{\operatorname{cond}\left(\lambda, C ; \Omega_{G V}\right)}{\operatorname{cond}\left(\lambda, K C K^{-1} ; \Omega_{G V}^{K C K^{-1}}\right)} \leq 3(n-2) \text {. }
$$

\section{Numerical experiments}

In this section, we will discuss briefly some numerical experiments that have been performed in order to confirm some of the results for eigenvalue condition numbers obtained in Sections 4,5 , and 6 . We have run several random numerical tests in MATLAB for comparing the unstructured componentwise condition number cond $(\lambda)$ in Theorem 2.8 and the structured ones $\operatorname{cond}\left(\lambda ; \Omega_{Q S}\right)$ and $\operatorname{cond}\left(\lambda ; \Omega_{G V}\right)$. We have started by generating the vectors

$$
\boldsymbol{l} \in \mathbb{R}^{n-2}, \boldsymbol{v} \in \mathbb{R}^{n-1}, \boldsymbol{d} \in \mathbb{R}^{n}, \boldsymbol{e} \in \mathbb{R}^{n-1}, \text { and } \boldsymbol{u} \in \mathbb{R}^{n-2},
$$

containing the randomly generated parameters of the tangent-Givens-vector representation in Definition 5.3 , by using the command randn from MATLAB. In addition, in some tests we have scaled the parameters in $\boldsymbol{v}$ and $\boldsymbol{e}$ as explained in (7.2). Then, we build the quasiseparable matrix $C$ of size $n \times n$ generated by these parameters and we obtain its eigenvalues and eigenvectors using the standard command eig from MATLAB. The parameters in $\Omega_{Q S}:=\Omega_{Q S}^{G V}$ in Definition 5.3 are also computed. Finally, we compute the structured eigenvalue condition numbers $\operatorname{cond}\left(\lambda ; \Omega_{Q S}\right)$ and $\operatorname{cond}\left(\lambda ; \Omega_{G V}\right)$, using our fast Algorithms 1 and 2 respectively, and the unstructured condition number $\operatorname{cond}(\lambda)$ using direct matrixvector multiplication with a resulting cost of order $2 n^{2}+\mathcal{O}(n)$ operations (note that cond $(\lambda)$ can also be computed in $\mathcal{O}(n)$ operations since $|C|$ is also a quasiseparable matrix), and we compare these three condition numbers.

When the generated parameters are completely random (i.e., no scaling has been introduced), we have not observed large differences between the eigenvalue condition numbers, i.e., $\operatorname{cond}(\lambda) \approx \operatorname{cond}\left(\lambda ; \Omega_{Q S}\right) \approx$ $\operatorname{cond}\left(\lambda ; \Omega_{G V}\right)$, and all of them are very often moderate.

On the other hand, as announced above, we have also performed tests where some scalings over the randomly generated parameters of the tangent-Givens-vector representation have been introduced in order to build an unbalanced quasiseparable matrix. After generating the random vectors as in (7.1), we have scaled the parameters in $\boldsymbol{v}$ and $\boldsymbol{e}$ by creating the vectors (using MATLAB standard notation):

$$
\boldsymbol{s c \boldsymbol { v }}=\left(10 .^{\wedge}(k:-(k-1) /(n-2): 1)\right) \text { and } \boldsymbol{s c e}=\left(10 .^{\wedge}(1:(k-1) /(n-2): k)\right),
$$

where $k$ is a fixed natural number not greater than 10 in our experiments, and considering

$$
\boldsymbol{v}=10^{2} * \boldsymbol{s c} \boldsymbol{v} . * \boldsymbol{v} \text { and } \boldsymbol{e}=10^{2} * \text { sce. } * \boldsymbol{e},
$$

which may produce unbalanced rows and columns in the lower and upper triangular parts of the matrix generated by these parameters (see Example 5.2). Therefore, we may expect large unstructured eigenvalue condition numbers. In this way, for different values of $k$ and different sizes $n$, we have found distributions of the tangent-Givens-vector parameters that produce $\{1 ; 1\}$-quasiseparable matrices such 
that the unstructured condition number $\operatorname{cond}(\lambda)$ is much larger than the structured ones, $\operatorname{cond}\left(\lambda ; \Omega_{Q S}\right)$ and $\operatorname{cond}\left(\lambda ; \Omega_{G V}\right)$. In fact, for $n=200$ and $n=300$, with $k=5$ in both cases, we have obtained particular matrices such that:

\begin{tabular}{|c|l|l|l|l|}
\hline$n$ & $\lambda_{\min }$ & $\lambda_{\max }$ & $\max _{\lambda}\left\{\frac{\operatorname{cond}(\lambda)}{\operatorname{cond}\left(\lambda ; \Omega_{Q S}\right)}\right\}$ & $\max _{\lambda}\left\{\frac{\operatorname{cond}\left(\lambda ; \Omega_{Q S}\right)}{\operatorname{cond}\left(\lambda ; \Omega_{G V}\right)}\right\}$ \\
\hline 200 & 555.2761 & $-2.4628 \cdot 10^{4}+1.6840 \cdot 10^{5} i$ & $4.7847 \cdot 10^{11}$ & 3.3337 \\
\hline 300 & 60.5936 & $-1.3533 \cdot 10^{5}+2.6060 \cdot 10^{5} i$ & $2.4201 \cdot 10^{10}$ & 3.2570 \\
\hline
\end{tabular}

where $\lambda_{\min }$ and $\lambda_{\max }$ denote the respective minimum and maximum eigenvalues in absolute value, while $\max _{\lambda}\left\{\frac{\operatorname{cond}(\lambda)}{\operatorname{cond}\left(\lambda ; \Omega_{Q S}\right)}\right\}$ and $\max _{\lambda}\left\{\frac{\operatorname{cond}\left(\lambda ; \Omega_{Q S}\right)}{\operatorname{cond}\left(\lambda ; \Omega_{G V}\right)}\right\}$ denote the respective maximum values of the quotients, both taken over the corresponding sets of the eigenvalues of the particular considered matrices for $n=200$ and $n=300$. Furthermore, if we denote by $\lambda_{\mathrm{opt}}$ the eigenvalue where the maximum of the quotient $\left\{\frac{\operatorname{cond}(\lambda)}{\operatorname{cond}\left(\lambda ; \Omega_{Q S}\right)}\right\}$ occurs, we have:

\begin{tabular}{|c|l|l|l|}
\hline$n$ & $\lambda_{\text {opt }}$ & $\operatorname{cond}\left(\lambda_{\text {opt }}\right)$ & $\operatorname{cond}\left(\lambda_{\text {opt }} ; \Omega_{Q S}\right)$ \\
\hline 200 & $-2.4569 \cdot 10^{3}+3.7791 \cdot 10^{2} i$ & $2.4780 \cdot 10^{13}$ & 51.7901 \\
\hline 300 & $-1.0088 \cdot 10^{4}$ & $1.8703 \cdot 10^{11}$ & 7.7281 \\
\hline
\end{tabular}

It is worth mentioning that, as one would expect, repeating these experiments for values of $k$ greater than 5 (which produce matrices with strongly unbalanced lower and upper triangular parts), we obtained similar results to the ones above.

These examples show not only that the structured eigenvalue condition number $\operatorname{cond}\left(\lambda ; \Omega_{Q S}\right)$ (and, therefore, $\operatorname{cond}\left(\lambda ; \Omega_{G V}\right)$ ) may be much smaller than the unstructured one cond $(\lambda)$, but also that there exist $\{1 ; 1\}$-quasiseparable matrices having eigenvalues that are very ill conditioned with respect to perturbations of its entries, but that are very well conditioned with respect to perturbations of its quasiseparable parameters.

In addition, as one would expect from Theorems 6.1 and 6.3, there is not much difference between the values of $\operatorname{cond}\left(\lambda ; \Omega_{Q S}\right)$ and $\operatorname{cond}\left(\lambda ; \Omega_{G V}\right)$, therefore we have omitted the corresponding column for the quotient $\frac{\operatorname{cond}\left(\lambda ; \Omega_{Q S}\right)}{\operatorname{cond}\left(\lambda ; \Omega_{G V}\right)}$ in the second table above. In fact, in all our tests we have obtained that

$$
\frac{\operatorname{cond}\left(\lambda ; \Omega_{Q S}\right)}{\operatorname{cond}\left(\lambda ; \Omega_{G V}\right)}<15
$$

which suggests that the bound in Theorem 6.3 may be improved up to a constant.

\section{Conclusions}

We have provided a general formula for the relative condition number of a simple eigenvalue of any matrix that can be represented by a set of parameters with respect to relative componentwise perturbations of these parameters. This result has been obtained by using differential calculus techniques and can be generalized to other conditioning problems. Based on this formula, we have obtained expressions for the eigenvalue condition numbers of $\{1 ; 1\}$-quasiseparable matrices with respect to relative componentwise perturbations of the parameters in the quasiseparable and the Givens-vector representations, and we have developed fast algorithms with cost $O(n)$ operations for computing these condition numbers. As far as we know, these results are the first ones available in the literature dealing with structured perturbations of low-rank structured matrices. Numerical tests comparing the new structured eigenvalue condition numbers with the unstructured componentwise condition number have been performed and have revealed that the eigenvalues of quasiseparable matrices may be very well-conditioned under relative perturbations of the parameters, but very ill-conditioned under general unstructured relative perturbations of the matrix entries. In contrast, it has been proved theoretically that the opposite cannot happen. Therefore, for $\{1 ; 1\}$-quasiseparable matrices, we have established that the structure should play a key role in the accuracy of eigenvalue computations since the sensitivity of their simple eigenvalues is potentially much smaller to perturbations of the parameters in the covered representations than to perturbations of the matrix entries. In addition, we have proved that all considered representations of $\{1 ; 1\}$-quasiseparable matrices are equivalent with respect to the eigenvalue sensitivity and that the Givens-vector representation is the one leading to the smallest eigenvalue condition numbers. 
Future lines of research related to the results in this paper include to develop and analyze structured eigenvalue condition numbers for other classes of low-rank structured matrices of higher ranks, as well as to analyze the structured sensitivity of other problems involving low-rank structured matrices. In particular, the authors are presently studying structured condition numbers for the solution of linear systems of equations whose coefficient matrices are quasiseparable.

Acknowledgements. The authors sincerely thank two anonymous referees and the Associate Editor handling this manuscript (Prof. Ilse Ipsen) for their extremely detailed readings of this long and technical paper, for correcting many grammatical typos, and for providing constructive and insightful comments that have contributed to a significant improvement of the original manuscript.

\section{References}

[1] Asplund, E., Inverses of matrices $a_{i j}$ which satisfy $a_{i j}=0$ for $j>i+p$, Math. Scand., 7:57-60, 1959.

[2] Asplund, S.O., Finite boundary value problems solved by Green's matrix, Math. Scand., 7:49-56, 1959.

[3] Aurentz, J.L., Mach, T., Vandebril, R., Watkins, D.S., Fast and backward stable computation of roots of polynomials, SIAM J. Matrix Anal. Appl., 36(3):942-973, 2015.

[4] Barlow, J.L., IPsen, I.C.F., Scaled Givens rotations for the solution of linear least squares problems on systolic arrays, SIAM J. Sci. Stat. Comput., 8(5):716-733, 1987.

[5] Bebendorf, M., Why finite element discretizations can be factored by triangular hierarchical matrices, SIAM J. Numer. Anal., 45(4):1472-1494, 2007.

[6] Bella, T., Olshevsky, V., Stewart, M., Nested product decomposition of quasiseparable matrices, SIAM J. Matrix Anal. Appl., 34(4):1520-1555, 2013.

[7] Berger, W.J., Saibel, E., On the inversion of continuant matrices, Frankl. Inst.-Eng Appl. Math., 6:249-253, 1953.

[8] Bindel, D., Demmel, J.W., Kahan, W., Marques, O., On computing Givens rotations reliably and efficiently, ACM Trans. Math. Softw., 28(2):206-238, 2002.

[9] Bini, D.A., Gemignani, L., Pan, V.Y., Fast and stable QR eigenvalue algorithms for generalized companion matrices and secular equations, Numer. Math., 100(3):373-408, 2005.

[10] Bini, D.A., Gemignani, L., Tisseur, F., The Ehrlich-Aberth method for the nonsymmetric tridiagonal eigenvalue problem, SIAM J. Matrix Anal. Appl., 27(1):153-175, 2005.

[11] Bini, D.A., Boito, P., Eidelman, Y., Gemignani, L., Gohberg, I., A fast implicit QR eigenvalue algorithm for companion matrices, Linear Algebra Appl., 432:2006-2031, 2010.

[12] Börm, S., Grasedyck, L., Hybrid cross approximation of integral operators, Numer. Math., 101:221-249, 2005.

[13] Börm, S., Grasedyck, L., Hackbusch, W., Introduction to hierarchical matrices with applications, Eng. Anal. Bound. Elemen., 27:405-422, 2003.

[14] Chandrasekaran, S., Gu, M., Xia, J., Zhu, J., A fast QR algorithm for companion matrices, Recent advances in matrix and operator theory, 111-143, Oper. Theory Adv. Appl., 179, Birkhäuser, Basel, 2008.

[15] Delvaux, S., Van Barel, M., A QR-based solver for rank structured matrices, SIAM J. Matrix Anal. Appl., 30(2):464-490, 2008.

[16] Dopico, F.M., Olshevsky, V., Zhlobich, P., Stability of QR-based fast system solvers for a subclass of quasiseparable rank one matrices, Math. Comp., 82:2007-2034, 2013.

[17] Eidelman, Y., Gohberg, I., On a new class of structured matrices, Integr. Equ. Oper. Theory, 34(3):293-324, 1999. 
[18] Eidelman, Y., Gohberg, I., On generators of quasiseparable finite block matrices, Calcolo, 42:187$214,2005$.

[19] Eidelman, Y., Gohberg, I., Haimovici, I., Separable Type Representations of Matrices and Fast Algorithms. Volume 1. Basics. Completion Problems. Multiplication and Inversion Algorithms, Operator Theory: Advances and Applications, 234. Birkhäuser/Springer, Basel, 2014

[20] Eidelman, Y., Gohberg, I., Haimovici, I., Separable Type Representations of Matrices and Fast Algorithms. Volume 2. Eigenvalue Method, Operator Theory: Advances and Applications, 235. Birkhäuser/Springer, Basel, 2014

[21] Eidelman, Y., Gohberg, I., Olshevsky V., The QR iteration method for Hermitian quasiseparable matrices of an arbitrary order, Linear Algebra Appl., 404:305-324, 2005.

[22] Ferreira, C., Parlett, B., Dopico, F.M., Sensitivity of eigenvalues of an unsymmetric tridiagonal matrix, Numer. Math., 122(3):527-555, 2012.

[23] Fiedler, M., Markham T.L., Generalized totally nonnegative matrices, Linear Algebra Appl., $345: 9-28,2002$.

[24] Gantmacher, F.R., Krein, M.G., Oscillation Matrices and Kernels and Small Vibrations of Mechanical Systems, AMS Chelsea Publishing, Providence, Rhode Island, 2002 (revised edition from the Russian original edition published in 1941).

[25] Geurts, A.J., A contribution to the theory of condition, Numer. Math., 39:85-96, 1982.

[26] Golub, G.H., Van Loan, C.F., Matrix Computations, 3rd ed., The Johns Hopkins University Press, Baltimore, 1996.

[27] Grasedyck, L., Kriemann, R., Le Borne, S., Parallel black box H-LU preconditioning for elliptic boundary value problems, Comput. Vis. Sci., 11:273-291, 2008.

[28] Higham, N.J., Accuracy and Stability of Numerical Algorithms, 2nd edn., Society for Industrial and Applied Mathematics, Philadelphia, 2002.

[29] Higham, D.J., Higham, N.J., Structured backward error and condition of generalized eigenvalue problems, SIAM J. Matrix Anal. Appl., 20(2):493-512, 1998.

[30] IPSEn, I.C.F., Relative perturbation results for matrix eigenvalues and singular values, Acta $\mathrm{Nu-}$ merica, 7:151-201, 1998.

[31] Karow M., Kressner D., Tisseur F, Structured eigenvalue condition numbers, SIAM J. Matrix Anal. Appl., 28(4):1052-1068, 2006.

[32] LI, R.C., Relative perturbation theory. III. More bounds on eigenvalue variations, Linear Algebra Appl., 266:337-345, 1997.

[33] Martinsson, P.G., Rokhlin V., A fast direct solver for boundary integral equations in two dimensions, J. Comput. Phys., 205:1-23, 2005.

[34] Martinsson, P.G., Rokhlin, V., Tygert, M., A fast algorithm for the inversion of general Toeplitz matrices, Comput. Math. Appl., 50:741-752, 2005.

[35] Parlett, B.N., Reinsch, C., Balancing a matrix for calculation of eigenvalues and eigenvectors, Numer. Math., 13:292-304, 1969.

[36] Rice, J.R., A theory of condition, SIAM J. Numer. Anal., 3:287-310, 1966.

[37] Roy, S.N., Sarhan A.E., On inverting a class of patterned matrices, Biometrika, 43:227-231, 1956.

[38] Stewart, G.W., Sun, J., Matrix Perturbation Theory, Academic Press, Boston, 1990.

[39] Van Barel, M., Vandebril, R., Van Dooren, P., Frederix, K., Implicit double shift QRalgorithm for companion matrices, Numer. Math., 116:177-212, 2010. 
[40] Vandebril, R., Van Barel, M., Mastronardi, N., A note on the representation and definition of semiseparable matrices, Numer. Linear Algebra Appl., 12:839-858, 2005.

[41] Vandebril, R., Van Barel, M., Golub G., Mastronardi, N., A bibliography on semiseparable matrices, Calcolo, 42:249-270, 2005.

[42] Vandebril, R., Van Barel, M., Mastronardi, N., Matrix Computations and Semiseparable Matrices. Volume 1. Linear Systems, The Johns Hopkins University Press, Baltimore, 2008.

[43] Vandebril, R., Van Barel, M., Mastronardi, N., Matrix Computations and Semiseparable Matrices. Volume II. Eigenvalue and singular value methods, The Johns Hopkins University Press, Baltimore, 2008.

[44] Wilkinson, J.H., The Algebraic Eigenvalue Problem, Oxford University Press, New York, 1965. 